September 4, 1991

\title{
STUDIES OF COMPLEX FRAGMENT EMISSION IN HEAVY ION REACTIONS
}

\author{
Principle Investigators: R.J. Charity and L.G. Sobotka \\ Departments of Chemistry and Physics \\ Washington University \\ St. Louis, MO 63130
}

\section{Progress Report}

\author{
Department of Energy Grant \\ DE-FG02-87ER40316 \\ for the period
}

September 1, 1990 through September 1, 1991 
$\mathrm{DOE} / \mathrm{ER} / 40316--5$

DE92 000532

\title{
STUDIES OF COMPLEX FRAGMENT EMIISSION IN HEAVY ION REACTIONS
}

\author{
Principle Investigators: R.J. Charity and L.G. Sobotka \\ Departments of Chemistry and Physics \\ Washington University \\ St. Louis, MO 63130
}

Progress Report
Department of Energy Grant
DE-FG02-87ER40316
for the period

September 1, 1990 through September 1, 1991

\section{DISCLAIMER}

This report was prepared as an account of work sponsored by an agency of the United States


Government. Neither the United States Government or assumes any legal liability or responsiemployees, makes any warranty, express or implied, or ansu information, apparatus, product, or bility for the accuracy, completeness, or usefulness of any ininge privately owned rights. Referprocess disclosed, or represents that its use would not infrice by trade name, trademark, ence herein to any specific commercial product, procstitute or imply its endorsement, recommanufacturer, or otherwise does not necessarly constiut or any agency thereof. The views mendation, or favoring by the United Stan do not necessarily state or reflect those of the and opinions of authors expressed herein do 


\section{FORWARD}

This document is a progress report for the period September 1, 1990 through August 31, 1991, for the work done on the Department of Energy Grant no. DE-FG02-87ER40316. We have made an effort not to duplicate material in our previous progress reports or to have extensive overlap with the reports of our collaborators. The period covered in the present report is the second year of a three-year grant approved in the fall of 1989. The companion document serves as the proposal for the work to be done in the third and final year of this grant.

\section{TABLE OF CONTENTS}

I. INTRODUCTION 2

II. SCIENTIFIC

A. High Energy Photon Production In a HI Collision

B. The Mechanism for the Disassembly of Excited ${ }^{16} \mathrm{O}$ Projectiles into Four Alpha Particles

C. The Disassembly of Excited ${ }^{28}$ Si Projectiles

D. Large Pre-Fission Multiplicities From Temperature Dependent Friction and Fission Barriers 18

E. Multiplicity Correlations $\quad 24$

F. Molecular Beam Induced Fusion 26

III. TECHNICAL

A. Dwarf Ball and Wall (DWB) 29

B. Mini Wall (MW) $\quad 30$

C. Computer and Local Data Acquisition Systems 32

IV. SCIENTIFIC AND TECHNICAL PERSONAL 33

A. Washington University Personal

B. Outside Collaborators

V. ABSTRACTS OF PUBLICATIONS FOR THE PERIOD

SEPTEMBER 1990 THROUGH SEPTEMBER 1991. 


\section{Introduction}

Our proposed work involves the study of intermediate energy heavy-ion nuclear reactions. This work has two foci. On the one hand, we desire to learn about the properties of nuclear matter under abnormal conditions, in this energy domain, predominately low densities. This purpose runs abreast of the second, which is the study of the relevant reaction mechanisms. The two objectives are inexorably linked because our experimental laboratory for studing nuclear matter properties is a dynamic one. We are forced to ask how nuclear matter properties, such as phase transitions, are reflected in the dynamics of the reactions. It may be that irrefutable information about nuclear matter (say the equation of state) will not be extracted from the reaction work. Nevertheless, we are compelled to undertake this effort not only because it is the only game in town and as yet we do not know that information cannot be extracted, but also because of our second objective. The process leads to an understanding of the reaction mechanism themselves and therefore to the response characteristics of finite, prehaps non-equilibrium, strongly interacting systems.

Our plan is: To study energy, mass, and angular momentum deposition by studying incomplete fusion reactions. To gain confidence that we understand how highly excited systems decompose by complete excitation energy reconstruction and by using particle-particle correlations to study the dynamics. To study the characteristics of the most violent collisions, those producing many large fragments, via excitation function studies. Finally to explore the relationship between high energy photon production, which has been ascribed to nucleon-nucleon scattering, to large fragment production, which is certainly collective in a nucleon sense.

These avenues of research share a common theme, large complex fragment production. It is this feature, more than any other, which distinguishes the intermediate energy domain. 


\section{Scientific}

\section{A. High Energy Photon Production in a Heavy-Ion Reaction[1]}

L.G. Sobotka, L. Gallamore, A. Chbihi, D.G. Sarantites, D.W. Stracener [WU], and W. Bauer, D.R. Bowman, N. Carlin, R.T. deSouza, C.K. Gelbke, W.G. Gong, S. Hannuschke, Y.D. Kim, W.G. Lynch, R. Ronningen, M.B. Tsang, and F. Zhu [MSU], and J. R. Beene, M.L. Halbert, and M. Thoennessen [ORNL]

There is considerable interest in understanding the mechanism for the generation of the high energy photons produced in intermediate energy heavy-ion reactions. This is due to the fact these photons could provide considerable insight into the early stages of the reaction dynamics because once created the photons leave the interaction region without rescattering. High energy photons can be produced by both nucleus-nucleus and nucleon-nucleon bremsstrahlung. Studies of the photon angular distribution and the dependence of the photon yield on the projectile and target charge indicate that nucleus-nucleus bremsstrahlung does not make a significant contribution to the high energy photon yield in heavy-ion collisions.

Photon emission from nucleon-nucleon collisions will occur throughout the nucleus-nucleus collision. The spectrum of photons will reflect the relative nucleon-nucleon collision velocity distribution. It is useful (and perhaps a bit simplistic) to consider the two extreme pictures. On the one hand, photons will be produced by the initial nucleon-nucleon collisions. On the other hand, after much of the collective translational kinetic energy has been damped, high energy photon production can be thought of as a statistical process from an equilibrated region of the interaction. Such a statistical mechanism implies multiple collisions, and therefore would be isotropic in the moving frame of the emitter. Futhermore, unless the deposited energy is strictly proportional to the number of nucleons which comprise the statistical emitter, the photon spectrum would exhibit a strong dependence on the extent of energy deposition. This deposited energy would also be reflected in the particle production. Therefore for the statistical process, with the exception noted above, one expects the photon spectrum to be harder for those coliisions which produce a larger particle multiplicity.

In the other extreme case, if the first (or first few) neutron-proton collisions are responsible for the hard photon yield, the photon angular distribution would be isotropic in the nucleonnucleon center-of-mass frame and the shape of the spectrum relatively insensitive to the ultimate energy damping. However, the yield of high energy photons would be expected to increase as the overlap of the projectile and target increases, simply reflecting more neutron-proton collisions. The spectral shape, which reflects the distribution of initial relative neutron-proton energies, would be expected to be only weakly dependent on impact parameter. (A weak dependence is expected because the most peripheral collisions will only lead to an overlap of the surface regions where smaller mean nucleon kinetic energies are found.)

A few experimental studies have shown that the inclusive angular distribution of the high energy photons is consistent with emission from the nucleon-nucleon center-of-mass frame. This observation provides a strong argument for a dominant leading particle production mechanism. However, conclusions based on inclusive angular distributions could be misleading in that they contain contributions from collisions at all impact parameters and thus different reaction types. 
It is possible that the inclusive angular distribution results from the superposition of the contributions of reactions with different admixtures of the leading versus statistical processes.

To remove the uncertainty mentioned above, we have studied the production of photons by characterizing the events, which produce the photons, by the forward and backward, light and heavy, charged particle production. This characterization is far more complete than that of any of the previous studies of high energy photon production in an intermediate energy heavy-ion reaction. The system studied is $65 \mathrm{MeV} / \mathrm{u}^{40} \mathrm{Ar}+{ }^{93} \mathrm{Nb}$. The rather complete charged particle production information allows us to address the question of whether both high energy photon production and particle multiplicity are measures of impact parameter.

The average intensity of the beam (from the K1200 at MSU) was 0.07 particle nanoamperes. This intensity was limited by counting rate considerations for the charged particle detection device, the Dwarf Ball and Wall, each capable of light ion identification ( $\mathrm{p}, \mathrm{d}, \mathrm{t},{ }^{3} \mathrm{He}$, and $\alpha$ ) and element identification for $Z<15$. The solid angle coverage of the Dwarf system was $85 \%$ of $4 \pi$. For the purpose of this analysis, the charged particles are characterized by whether they are light $(Z<3)$ or heavy $(Z \geq 3)$ and by their emission angles. As we have done before, the angular region is coarsely, and rather arbitrarily, divided into that spanned by the forward Wall ( $\theta_{\text {lab }}<$ $35^{\circ}$ ) and the more backward angles covered by the Ball.

The photons were detected in two $\mathrm{BaF}_{2}$ arrays, each consisting of 19 close packed detectors. The individual $\mathrm{BaF}_{2}$ detectors have hexagonal cross sections of $36.6 \mathrm{~cm}^{2}$ and are $20 \mathrm{~cm}$ long. The two arrays were positioned, one on top of the other, $50 \mathrm{~cm}$ from the target, at $90^{\circ}$ to the beam. The detectors were individually calibrated with sources (extending up to $2.62 \mathrm{MeV}$ ) and the cosmic ray peak, the data for which were acquired during a 133 minute beam off run. The most probable energy deposition for a cosmic-ray was calculated with the simulation code GEANT. The results of GEANT have been verified to give the correct cosmic-ray energy deposition in a separate experiment where the $\mathrm{BaF}_{2}$ detectors were calibrated using the $15.1 \mathrm{MeV}$ photon produced in the reaction ${ }^{12} \mathrm{C}\left(\mathrm{p}, \mathrm{p}^{\prime}\right)$. Neutron pulses were distinguished by pulse shape discrimination. (The time of flight information was also used to reject neutron pulses, but this technique was only effective for pulses corresponding to deposited energies of less than $15 \mathrm{MeV}$, which are not of interest for the present study.) Each array of $19 \mathrm{BaF}_{2}$ detectors was treated as one detector after energy calibration and neutron rejection. (If any of the 19 individual detectors recorded a neutron pulse the sum information from the entire pack was rejected.) Each pack subtended $2.1 \%$ of $4 \pi$. Cosmic-ray pulses proved to be negligible, primarily due to the coincidence resolving time.

The charged particle multiplicity distributions are shown in Fig. IIA1a. This figure shows how the total multiplicity (thickest line) is composed of light ions (thin lines) and heavy ions (lines of intermediate thickness), emitted to forward and backward angles. The overwhelming majority of the multiplicity is due to the light ions, with the contribution from angles larger than $35^{\circ}$ exceeding that measured forward of this angle. On the other hand, the much smaller heavy-ion contribution, is forward focussed. Coincidence summing, primarily in the most forward detectors, reduces these measured multiplicities. This effect is small except for the largest muitiplicities, where it distorts the distribution. The photon energy spectra acquired in coincidence with various multiplicity bins of light and heavy, forward and backward charged particles are shown in Figs. IIA1b-1f. The most striking feature of these spectra is that the spectral shape is very nearly the same for all the gating conditions. Beyond this, we observe that in all cases, the larger the particle multiplicity, the larger the differential photor multiplicity. However, while the above is true, the magnitude of the increase varies considerably depending on the particle selection condition. 


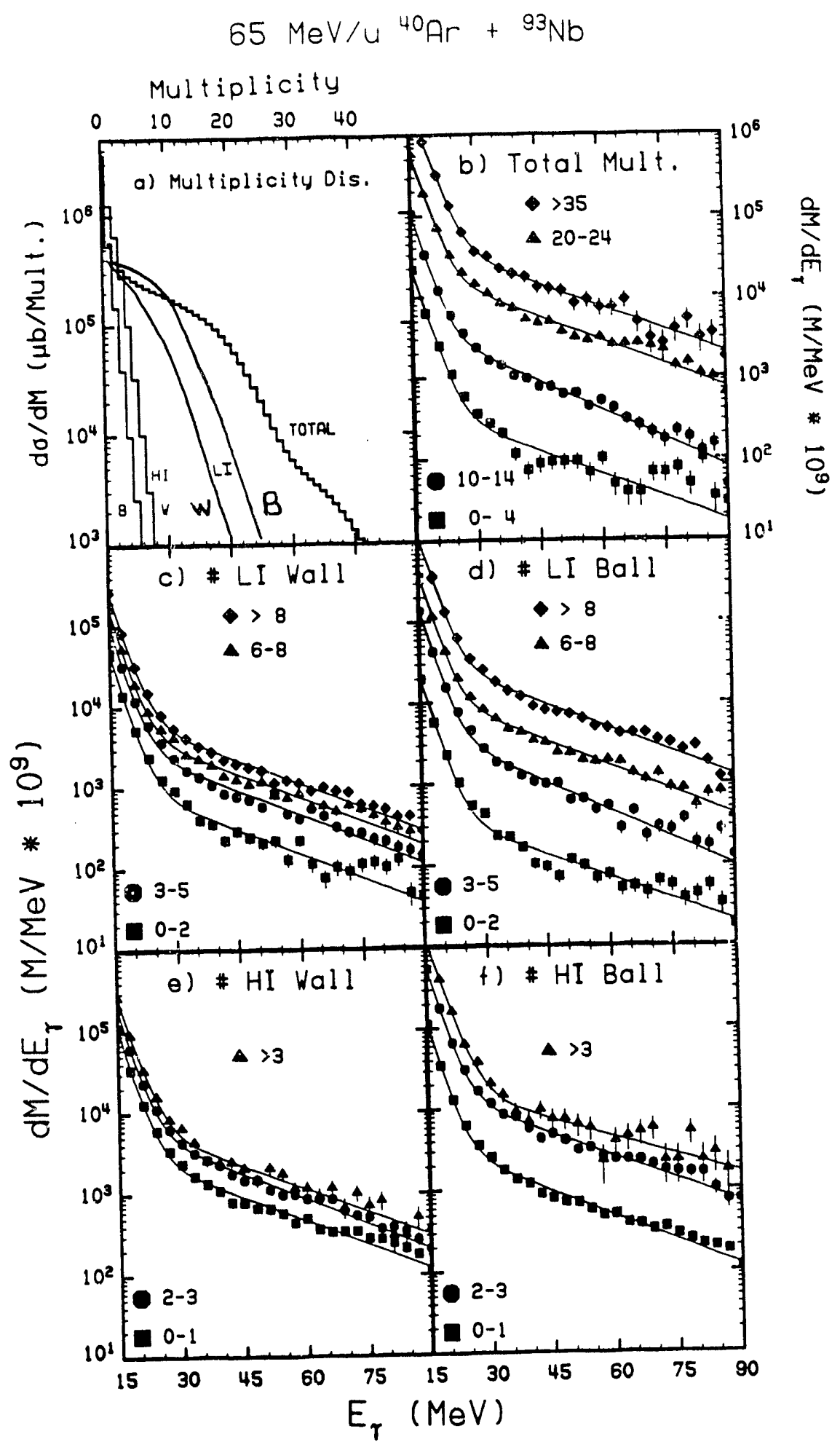

Fig. IIA1: a) Total charged particle multiplicity (thickest line) and its composition in terms light ions (thin lines) and heavy ions (intermediate thickness lines) for the Wall (W) and the Ball (B). The Wall subtends angles between approximately $5^{\circ}$ and $35^{\circ}$, while the Ball subtends the backward angles to approximately $170^{\circ}$. The photon spectra gated on selected bins of: b) total charged particle multiplicity, c) forward light-ion multiplicity, d) backward light-ion multiplicity, e) forward heavy-ion multiplicity, and f) backward heavy-ion multiplicity. The charged particle multiplicity bins are indicated in the figure. The ordinate is differential multiplicity ( $\mathrm{Mult} . / \mathrm{MeV}$ ), calculated assuming isotropic emission. 
For both light and heavy ions, a large charged particle multiplicity selection for the more backward angles (Ball) is far more effective at increasing the probability of high energy photon production then a similar selection on forward multiplicity (Wall). This is most clearly seen in the case of light ions, (compare Figs. IIA1c and IIA1d). In our previous work we have shown that large backward multiplicity is the best single indicator of linear momentum transfer. Therefore this figure illustrates that large momentum transfer events not only copiously produce fragments but also produce the most high energy photons.

In order to get the actual photon energy distribution and the correct absolute photon mutiplicity, we have unfolded the photon spectra. The response of the individual $\mathrm{BaF}_{2}$ packs was simulated with GEANT for photons generated $55 \mathrm{~cm}$ from the front face. The experimental spectra were fitted by sums of exponential functions (lines in Figs. IIA1b-1f) and the fits where unfolded. A sample of this procedure is shown in Fig. IIA2. The fit (thin line) represents the raw data quite well. The unfolding procedure (the result of which is indicated by the thick line in Fig. IIA2) increases the absolute multiplicity, in this high energy region, as well as making the spectrum slightly harder.

Unfolded spectra are shown as solid symbols in Fig. IIA3 for gates on the total multiplicity. (As these spectra result from the unfolding of fits to the raw data, no error bars are assigned to the individual points. A reasonable estimate for the uncertainty of the unfolded spectrum would be given by a region enveloped by symbols approximately $1 / 2$ the size of those in the figure at the low energy end to twice the size of the symbols at the high energy end.) The photon yield in the high energy region, $\left(E_{\gamma}>30 \mathrm{MeV}\right.$ ) is well described by a single exponential with an inverse slope parameter of $E_{o}($ data $)=23.9 \pm 3.7 \mathrm{MeV}$ and $25.3 \pm 2.4 \mathrm{MeV}$ for the data gated on the total multiplicity (Figs. IIA3) and the data gated on backward light-ion multiplicity (not shown), respectively. In both of these cases, the inverse slope parameter exhibits no statistically significant trend with particle multiplicity. The previous work on the substantially lighter system ${ }^{36} \mathrm{Ar}+$ ${ }^{27} \mathrm{Al}$ at $85 \mathrm{MeV} / \mathrm{u}$ observed a $40 \%$ increase in the inverse slope paramenter for central collisions as compared to the most peripheral. Our particle multiplicity sorted data are inconsistent with such a large change.

The similarity of the photon energy distribution for the different gates on multiplicity suggests that the fundamental neutron-proton bremsstrahlung spectrum is similar for all events and simply that the number of neutron-proton collisions increases with violence, as measured by the particle multiplicity. As mentioned previously, this is the trademark of a leading particle collision production mechanism. In such a picture, the spectrum is mainly determined by the initial relative nucleon-nucleon velocity distribution, which is only weakly dependent on geometry, while the probablity is proportional to the collision frequency, which is primarily determined by geometry.

To make quantitative statements about the production mechanism, detailed comparisons with model calculations are required. We have made such a comparison using the Boltzmann-UehlingUhlenbeck (BUU) equation. This treatment describes the time development of the system by a transport equation for the one-body density matrix, derived from the Bogoliubov, Born, Green, Kirkwood, Yvon (BBGKY) hierarchy. In order to make this comparison, we have converted the multiplicity scale to an impact parameter scale. We assume that the charged particle multiplicity increases monotonically with decreasing impact parameter. The interaction radius, below which reactions start and the detection device is sensitive, is taken as $R_{i n t}=11.75 \mathrm{fm}$. The calculations for the impact parameters corresponding to the mean multiplicity of the multiplicity bins are included in Fig. IIA3 as open symbols. 


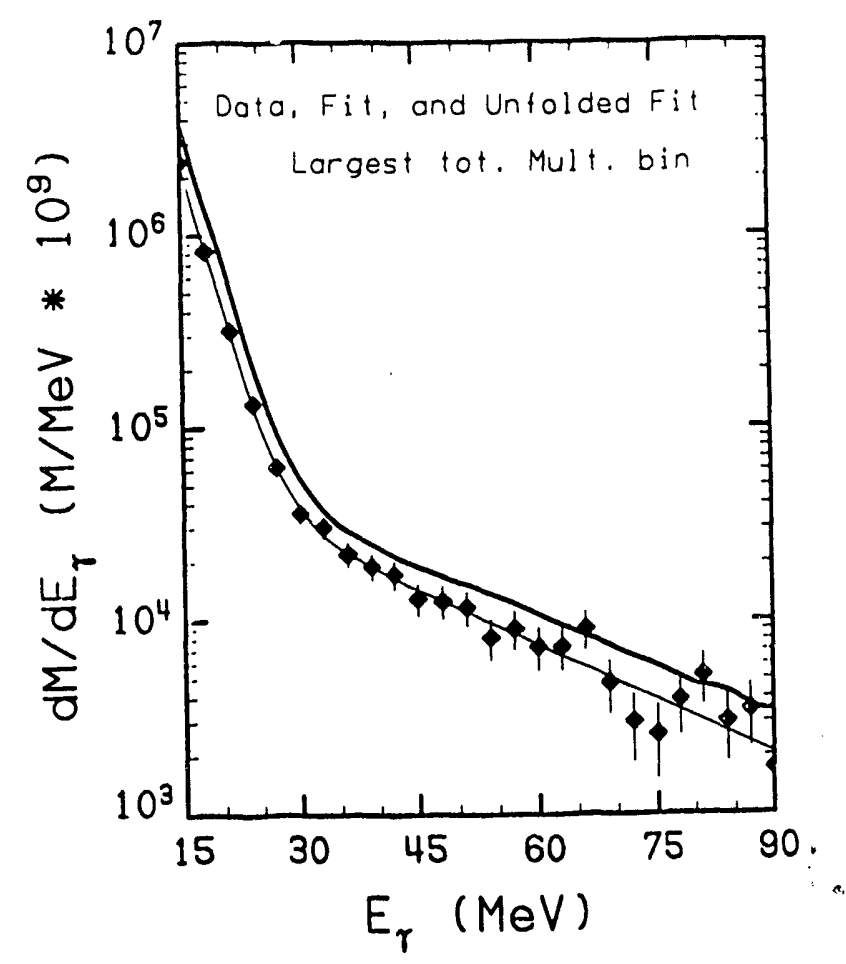

Fig. IIA2: Shown are the data (diamonds), fit of the data (thin line), and the spectrum resulting from the unfolding of the fit (thick line) for events with a total charged particle multiplicity greater than 35 .

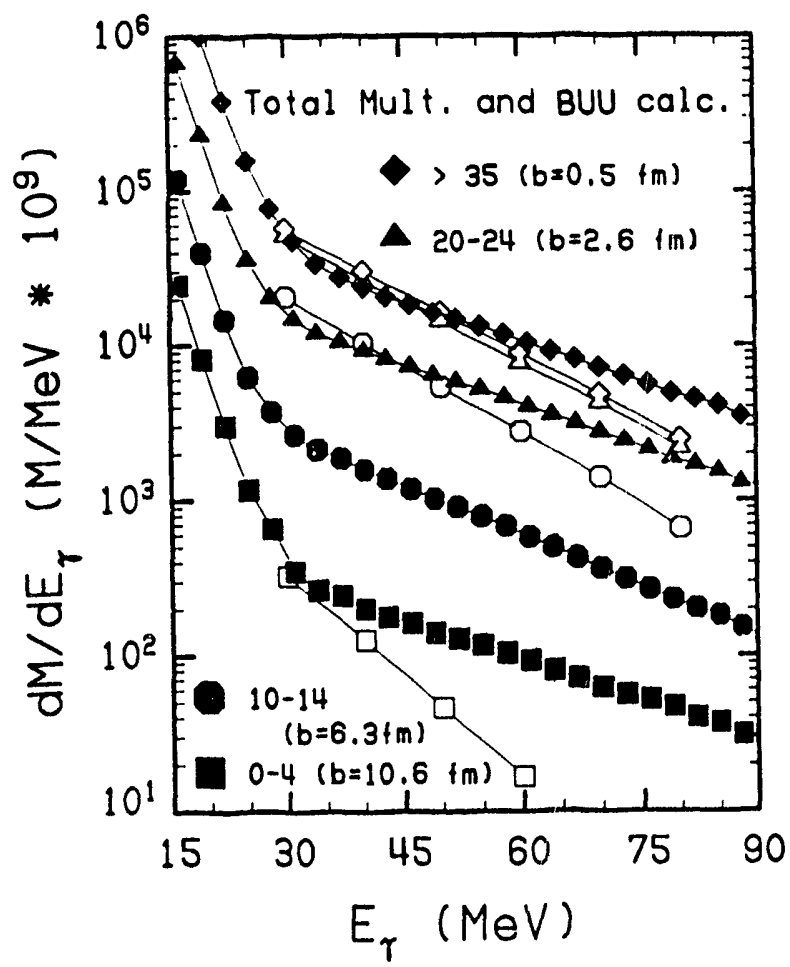

Fig. IIA3: Unfolded spectra are shown as solid symbols for 4 bins on the total charged particle multiplicity. BIU calculations at the corresponding impact parameters are shown as open symbols. 
The overall yield of the high energy photons, which is determined by the most central collisions, is well reproduced. However, the calculations underestimate the inverse slope parameter $\left(\mathrm{E}_{o}(\mathrm{BUU})=15.5 \pm 0.5 \mathrm{MeV}\right.$ for all impact parameters except the largest $)$ and fail to reproduce the regular decrease in the photon yield with decreasing paiticle multiplicity.

The underprediction of the inverse slope parameter suggests that either the extreme components of the momentum distributions of the colliding nuclei are underestimated or that the elementary photon production cross sections for large relative nucleon energies is underestimated. The second discrepancy, that the decrease of the photon yield with impact parameter (calculations) does not reproduce the observed trend with particle multiplicity, is illustrated in Fig. IIA4 where the integrated photon multiplicity is plotted versus impact parameter (a deduced parameter in the case of the data). The calculated yield follows the overlap volume of the colliding partners (solid line) while the data exhibit an exponential decline with the deduced impact parameter. While this difference could result from a deficiency in the calculation, it may also be due an inability to simply relate impact parameter to particle multiplicity. It is reasonable to expect that there exists a broad particle multiplicity distribution (reflecting a broad thermalized energy distribution) for a fixed impact parameter. In this case, one can only expect a comparison between a calculation (at fixed impact parameter) and experimental data (at fixed multiplicity) to be valid on the average, if the quantity being studied is not correlated directly to the particle production mechanism. If there was a direct correlation between high energy photon production and charged particle production, then the high energy photon production would track the charged particle multiplicity in a fashion which would not be given by a calculation which ignores the fluctuations and relates an average particle multiplicity to an average impact parameter. This is exactly what is suggested by the data and calculations shown in Figs. IIA3 and 4. At lower energies, deeply inelastic collisions provide an example of such a correlation. The impact parameter does not uniquely determine the energy loss, but given an energy loss the number of evaporated particles and the hardness of the statistical photon spectrum will track one another. In the energy domain of the present work, where statistical photon emmision does not appear to play a significant role, a plausible physical argument for such a correlation also exists, as long as the photon energy is small compared to the total thermalized energy. The violent nucleon-nucleon collisions which produce high energy photons are efficient at mixing the momentum components, decreasing the momentum components along the beam direction and generating transverse components. There ${ }^{\text {s }}$ re the leading neutron-proton scattering fosters the development of phase space distributions which appear thermal, thus increasing the multiplicity of particles which will be evaporated at a later stage of the reaction. Needless to say, if the photon energy is a significant part of the total thermalized energy, energy conservation will require an anticorrelation between photon and particle production. As a final comment, the presence of strong particle multiplicity fluctuations at fixed impact parameter may also explain why photon spectra gated on different multiplicity regions have indistinguishable inverse slope parameters.

In summary, we have made a detailed study of the charged particle multiplicity dependence of high energy photon production in the reaction $65 \mathrm{MeV} / \mathrm{u}{ }^{40} \mathrm{Ar}+{ }^{93} \mathrm{Nb}$. While the photon yield does increases dramatically with charged particle multiplicity, the shape of the spectrum remains essentially constant. This observation provides support for the interpretation that the high energy photon production in intermediate energy collisions result from nucleon-nucleon collisions early in the nucleus-nucleus collision dynamics. Calculations based on the BoltzmannUehling-Uhlenbeck equation for the time development of the one-body phase-space distribution and photon production via incoherent nucleon-nucleon collisions reproduce the overall photon 
yield but underestimate the slope ard fail to reproduce the trend of the differential photon multiplicity with charged particle multiplicity. The first difference is likely a failure of the calculation and suggests that more attention must be paid to the tails of the momentum components. The second difference may result from particle multiplicity fluctuations, at fixed impact parameter, which invalidate direct comparisons between data at fixed particle multiplicity and calculations at a fixed impact parameter.

\section{References}

[1] Condensed from a manuscript submitted for publication.

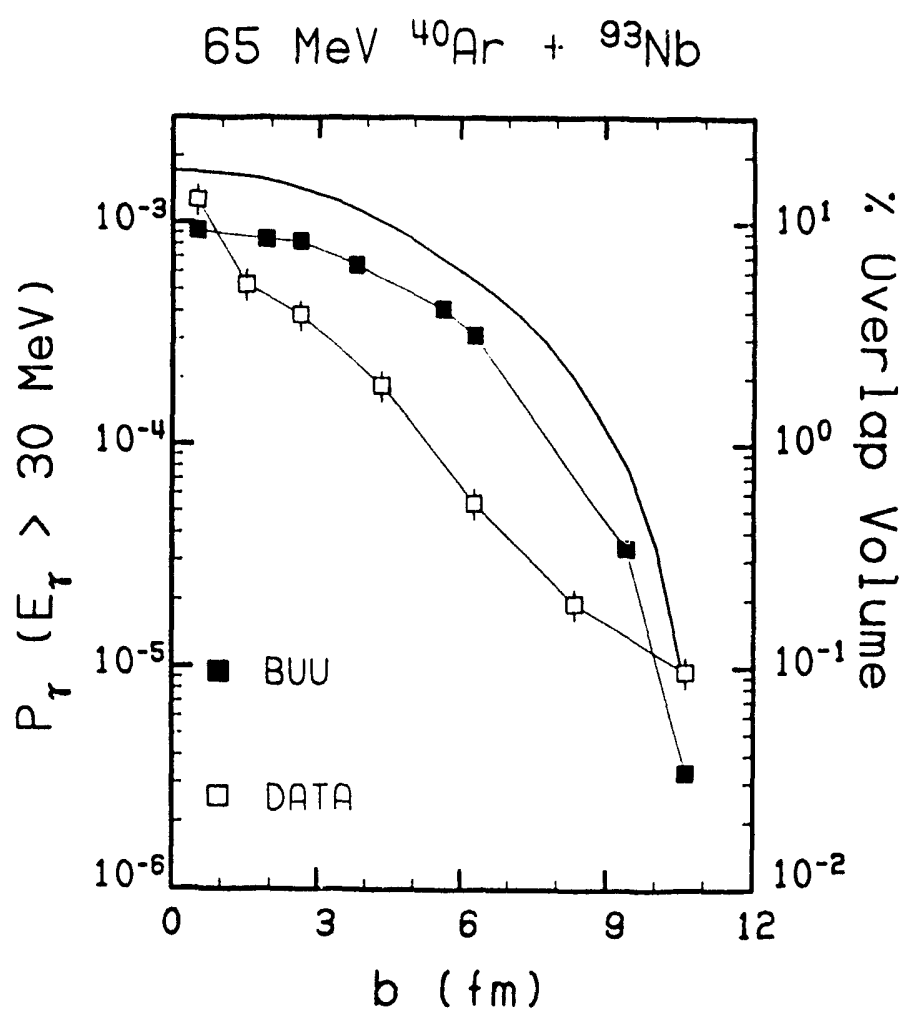

Fig. IIA4: Integrated yield of photons with energy exceeding $30 \mathrm{MeV}$. The data are show $\mathrm{n}$ as open symbols and are plotted as a function of a deduced impact parameter. The BUU reaction symulations are shown as solid symbols. The solid line is the percent overlap of two spheres averaged over straight line trajectories. 


\section{B. The Mechanism for the Disassembly of Excited ${ }^{16} \mathrm{O}$ Projectiles into Four Alpha Particles.[1]}

J. Barreto, R.J. Charity, L.F. Sobotka, D.G. Sarantites, D.W. Stracener, A. Chbihi, N.G. Nicolis [WU], R. Auble, C. Baktash, J.R. Beene, F. Bertrand, M. Halbert, D.C. Hensley, D.J. Horen, C. Ludemann, M. Thoennessen, and R. Varner [ORNL].

The decay of nuclear matter into many pieces is a topic of much interest at present. Models proposed to explain the disintegration process include both prompt breakup and sequential emission of the fragments. It is important in studies of many fragment events to find clear signatures for the different modes of disassembly. One useful technique is to study the relative velocities or relative angles between the fragments. If the fragments produced by the disassembly process are initially in close proximity and their subsequent interaction is dominated by their mutual Coulomb repulsions, then one expects a suppression of events with small relative velocities and relative angle. On the other hand, for a sequential process, if the time steps between emissions are sufficiently long, then the emitted fragments will not experience any mutual interactions. Small relative velocities will therefore not be suppressed. Analysis of relative velocities of intermediate mass fragments produced in central collisons have been performed by Trockel et al.[2]. Harmon et al. ${ }^{[3]}$ have measured relative angles of alpha particles produced in the disassembly of Oxygen projectiles. Both these studies conclude that the fragments are produced by a sequential decay mechanism.

In this work, we also report on a study of the breakup of ${ }^{16} \mathrm{O}$ projectiles into the four alpha particle exit channel. The projectiles, with bombarding energies of $\mathrm{E} / \mathrm{A}=25 \mathrm{MeV}$, were excited through peripheral interactions with a ${ }^{159} \mathrm{~Tb}$ target nuclei. The experiment was performed with an almost $4 \pi$ coverage of solid angle. The alpha particles produced by the disassambly of the excited projectile were detected in the Dwarf Wall CsI array and light charged particles emitted by the excited target nucleus were detected in the Dwarf Ball CsI array. Candidate ${ }^{16} \mathrm{O} \rightarrow 4 \alpha$ events were selected by requiring thai four, and only four, alpha particles were detected in the Wall array $\left(4 \lesssim \theta_{i a b} \lesssim 32^{\circ}\right)$.

If the primary reaction channel for correctly identified candidate events contains just two bodies, then all the particles detected in the Ball array must originate from the decay of the excited target nuclei. Only $\approx 20 \%$ of the candidate $4 \alpha$ projectile fragments are in coincidence with charged particles detected in the Ball counters. This is not surprising since the dominant decay mode of the target nuclei is expected to be neutron evaporation. The target nucleus is expected to be moving very slowly in the laboratory frame and hence the angular emission of these particles should thus be approximately isotropic in this frame. The experimental angular distributions for these charged particles are displayed in Fig. IIB1. Rather than the expected nearly flat behavior, these angular distributions are all strongly forward peaked and are not consistent with evaporation from the target nucleus. However, for angles greater than $90^{\circ}$, the distributions are significantly flatter.

The angular distributions thus indicate that either some of the candidate events are incorrectly identified or the interaction between the target and projectile nuclei is more complex than assumed. In either case, it is useful to try to reject such events. Events in which a charged particle is detected in one of the forward Ball counters $\left(\theta_{l a b}<90^{\circ}\right)$ are more likely to be of this nature; consequently all such events were removed from the sample of candidate events. 


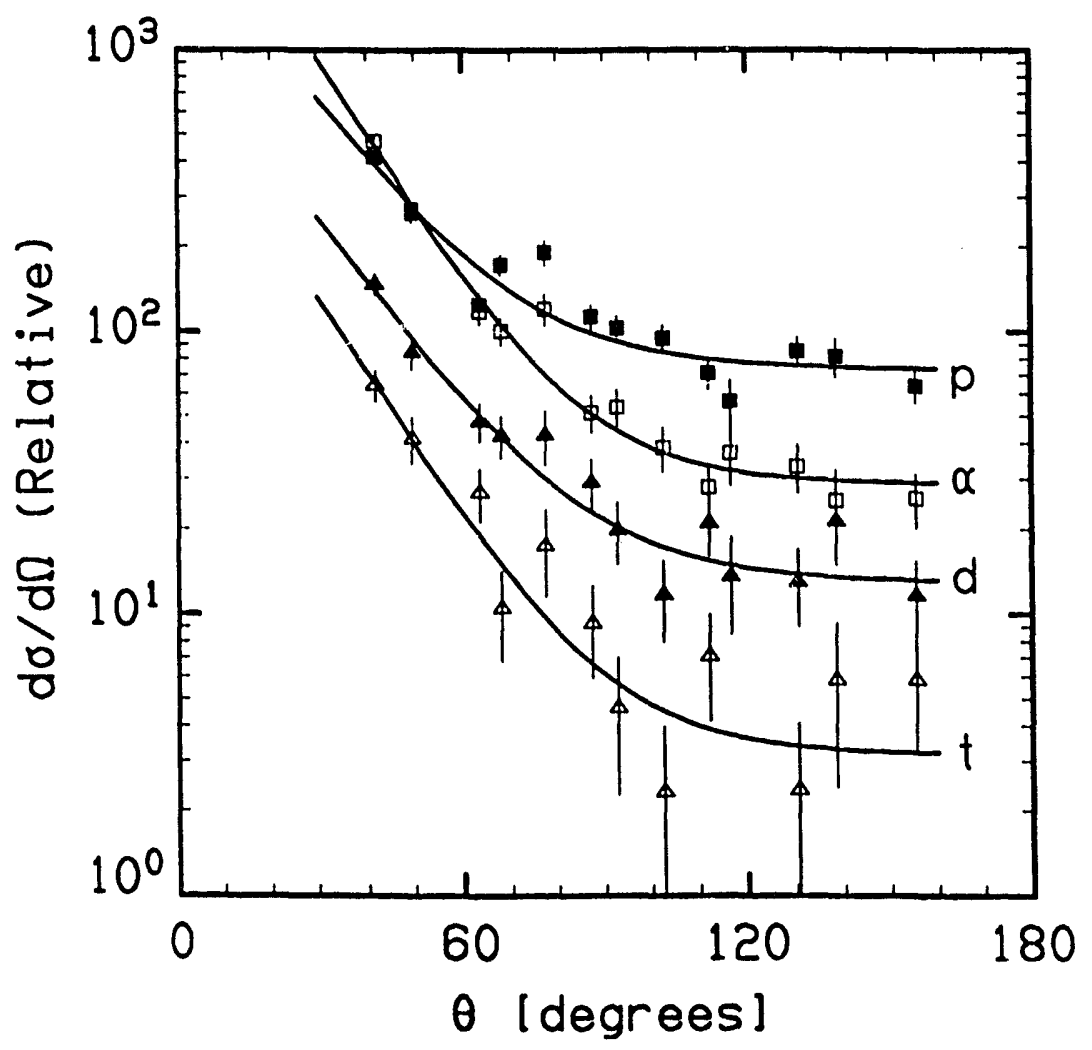

Fig. IIB1: Relative laboratory angular distributions of protons, deuterons, tritons and alpha particles detected in the Ball counters for events where four, and only four, alpha particles were detected by the Wall counters. The curves guide the eye. 
Note that this condition does not greatly reduce the number of candidate events as most candidate events were not in coincidence with any charged particle detected in the Ball counters.

As a further check as to the level of incorrectly identified candidate events, the magnitude of the particle multiplicities detected in the Ball counters were compared to the expectation for target evaporation. Figure IIB2 shows the detected mean multiplicities of protons and alpha particles as a function of the reconstructed target excitation energy. These multiplicities show a - apid increase with increasing target excitation energy as expected for evaporation. The mean multiplicities calculated with a statistical model calculation are indicated by the solid curve in Fig. IIB2. These calculations were corrected for the efficiency of the Dwarf Ball array using Monte Carlo simulations and the predicted mean multiplicity detected in the backward half of the Ball array is indicated by the dashed curve. The agreement between these multiplicities is quite good for low target excitation energies, but at the higher excitation energies the predicted multiplicities are larger than the values for the candidate events. This may indicate a problem with event selection or with the statistical model predictions at the higher excitation energies. However, the region of disagreement occurs only for the tail of the reconstructed target excitation energy distribution. For the bulk of the data the predicted and measured multiplicities are in agreement.

Events where an alpha particle evaporated from the target nucleus or from some intermediate source is incorrectly identified as one of the four alpha particles originating from the projectile should lead to low values of the reconstructed projectile velocity as such alpha particles have velocities much less than the beam velocity. Incorrect identification should therefore be a greater problem at the larger kinetic energy dissipations and thus larger reconstructed target excitation energies. This may explain the deviation of the measured and predicted multiplicities at large excitation energies in Fig. IIB2. As an extra criterion for removing such events, the effect of insisting that all the candidate alpha particles have laboratory velocities greater than $4.5 \mathrm{~cm} / \mathrm{ns}$ ( $65 \%$ of the beam velocity) was investigated. This velcoity is greater than typical values associated with target evaporation. This extra condition completely removed the tail of the target excitation energy distribution above $150 \mathrm{MeV}$ for which the predicted and measured multiplicities were in disagreement.

The experimental relative angle distribution was compared to those predicted with the Monte Carlo simulations of sequential evaporation and prompt decay mechanisms. In the sequential decay simulation, alpha particles were evaporated isotropically. The Weisskopf evaporation formulism was used to choose the kinetic energies of the alpha particles. No interactions between the emitted alpha particles was considered. For the prompt simulation, the approach used by López and Randrup ${ }^{[4]}$ was followed. Initial positions of the alpha particles were chosen at random inside a volume larger than that for a cold ${ }^{16} \mathrm{O}$ nucleus. The trajectories of the alpha particles under their mutual Coulomb interactions were then followed until the velocity vectors approached their asymptotic values.

Three otier simulations were also performed. Firstly a fission simulation where the ${ }^{16} \mathrm{O}$ projectile fissions : nto two ${ }^{8} \mathrm{Be}$ nuclei, each of which sequentially decay's into two al pha particles. This process was simulated using the same evaporation logic as described for the sequential evaporation mechanism. Secordly, a prompt-sequential simulation where the ${ }^{16} \mathrm{O}$ promptly breaks up into two alpha particles and a ${ }^{8} \mathrm{Bc}$ which later decays. Lastly, a random simulation was performed; four alpha particle velocity vectors were chosen randomly within a sphere in velocity space of arbitrary radius. The center of mass velocity of the particles was calculated and subtrasted from these velocity vectors so that the total momentum was zero. It should be noted that the initial 
distribution of relative angles in the prompt simulations was exactly the same as for this random simulation; the differences in the final distributions reflect the effect of the Coulomb interaction as the alpha particles move away from each other.

The simulated events were passed through the detector filter and the relative angles were reconstructed as for the experimental data. For each event, there are six possible relative angles. These angles are taken with respect to the center of mass of the four alpha particles. The joint distribution of relative angles predicted by each of the simulations have the same overall shape. Differences between the distributions can be highlighted by dividing each distribution by one such representative distribution. To this end, the experimental and simulated relative angle distributions were divided by the distribution obtained with the random simulations.

Figure IIB3a compares the resultant experimental and simulated distributions for all candidate events. In Fig. 3b only candidate events where all four alpha particles have a laboratory velocity greater than $4.5 \mathrm{~cm} / \mathrm{ns}$ were used to produce the experimental and simulated distributions. These events are less likely to be contaminated by incorrectly identified events; however the shapes of the experimental distributions for the two criteria are identical within the statistical errors.

The experimental relative angle distributions in Fig. IIB3a and $3 \mathrm{~b}$ are in good agreement with the predictions of the random simulation which correspond to a constant value of unity in these plots. The sequential evaporation predictions, indicated by the thick solid curve, differ from that of the random simulation by at most $3 \%$ and are also in good agreement with the experimental data. The suppression of small relative angles predicted by the prompt simulation is most clearly seen in these plots. Its predictions, indicated by the thin solid curve, show a suppression of up to $\approx 60 \%$. No such suppression is seen in the experimental data which are clearly in disagreement with the predictions of this simulation at all relative angles. The promptsequential simulation also predicts a suppression at smaller relative angles, as shown by the long dash curve in Figs. IIB3a and 3b, but of much smaller magnitude than for the prompt simulation. These curves are also inconsistent with the experimental data. Finally, the predictions of the fission simulation, indicated by the short dashed curve, fail to reproduce the experimental data at medium and large relative angles.

Within the statistical error of the measurements, the relative angles between the four alpha particles are random within the constraint of momentum conservation. They are also in excellent agreement with the expectation for the sequential evaporation decay mechanism. The prompt decay of the excited projectile into four alpha particles can be ruled out as the prominent decay mechanism leading to the $4 \alpha$ exit channel. There is no evidence for any suppression of small relative angles due to Coulomb interactions between the alpha particles. Also the mechanism of fission can be ruled out as an important decay channel of the projectile, although in the statistical model one would expect some small probability for this decay mode. The prompt decay of the projectile into two alpha particles and $\mathrm{a}^{8} \mathrm{Be}$ is also ruled out as an important decay mode. In conclusion, for the range of projectile excitation energies sampled in this study ( $\mathrm{E}^{*} /$ nucleon $\lesssim 4.5 \mathrm{MeV}$ ), the data indicate that the four alpha exit channel is produced predominantly by the sequential evaporation of these particles.

\section{References}

[1] Condensed from a manuscript submitted for publication. 
[2] R. Trockel, U. Lynen, J. Pochodzalla, W. Trautmann, N. Brummund, E. Eckert, R. Glasow, K.D. Hildenbrand, K.H. Kampert, W.F.J. Müller, D. Pelte, H.J. Rabe, H. Sann, R. Santo, H. Stelzer, and R. Wada, Phys. Rev. Letts. 59, 2844 (1987).

[3] B.A. Harmon, J. Pouliot, J. López, J. Suro, R. Knop, Y. Chan, D.E. DiGregorio, and R.G. Stokstad, Phys. Letts. B235, 234 (1990).

[4] J.A. López and J. Randrup, Nucl. Phys. A491, 477 (1989).
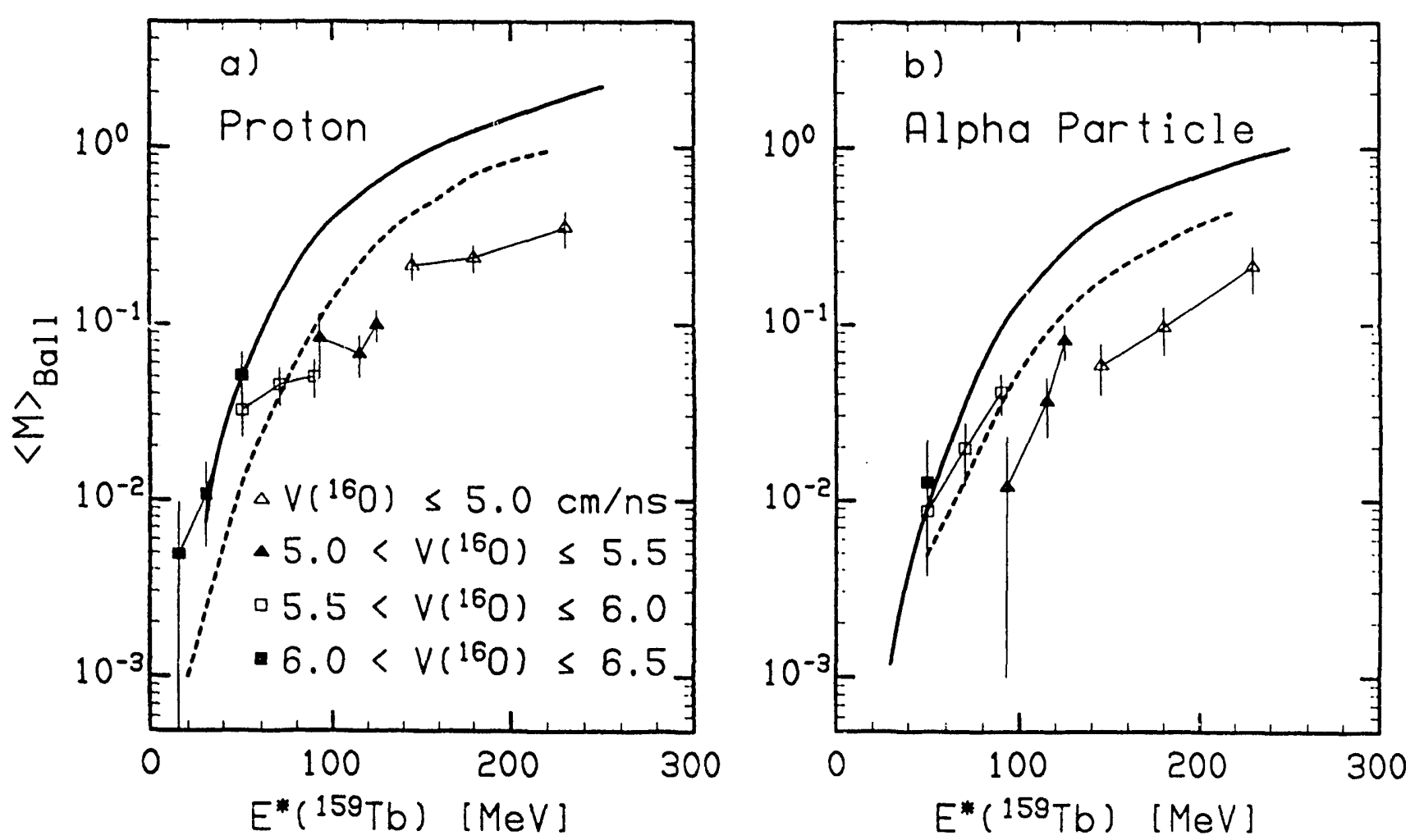

Fig. IIB2: Multiplicities as a function of the reconstructed target excitation energy of a) protons and b) alpha particles detected in the backward hemisphere of the Ball for events where foir, and only four, alpha particles were detected by the Wall counters and no other particles were detected in the forward hemisphere of the Ball. The solid curves indicate statistical model predictions of the proton and alpha particle multiplicities evapnrated from the target nucleus. The dashed curves indicate the predicted multiplicities once the effect of the incomplete detector angular coverage and the selection criteria are considered. 




Fig. IIB3: Relative angle distributions $\mathrm{P}(\beta)$ divided by the corresponding predictions obtained from the random simulation $\mathrm{P}_{\text {random }}(\beta)$ for a) all selected ${ }^{16} \mathrm{O} \rightarrow 4 \alpha$ events and $\mathrm{b}$ ) events where all four alpha particles have laboratory velocities greater than $4.5 \mathrm{~cm} / \mathrm{ns}$. The experimental results are represented by the data points with error bars. The predictions of the sequential evaporation simulation are indicated by the thick solid curve, the prompt simulation by the thin solid curve, the prompt-sequential by the long dashed curve, and the fission simulation by the short dashed curve. 


\section{The Disassembly of Excited ${ }^{28}$ Si Projectiles}

R.J. Charity, J. Barreto, L.G. Sobotka, D.G. Sarantites, D.W. Stracener, A. Chbihi, N.G. Nicolis [WU], D.C. Hersley, J.R. Beene, and M. Halbert, [ORNL].

The investigation of the disassembly mechanisms of excited projectiles has been continued with the study of the reaction $\mathrm{E} / \mathrm{A}=25 \mathrm{MeV}{ }^{28} \mathrm{Si}+{ }^{100} \mathrm{Mo}$. Projectile exit channels such as ${ }^{16} \mathrm{O}+3 \alpha$ and ${ }^{12} \mathrm{C}+4 \alpha$ have been studied so far. Unlike the ${ }^{16} \mathrm{O} \rightarrow 4 \alpha$ study, the reconstructed projectile excitation energies for these exit channels are quite localized in excitation energy. This can readily be understood: The lower threshold in excitation energy corresponds to the binding energy plus Coulomb energy for the channel. A higher threshold occurs because when ${ }^{16} \mathrm{O}$ or ${ }^{12} \mathrm{C}$ fragments are produced with high enough excitation energies they also decay by particle emission. The angular distributions of particles detected in the ball array in coincidence with with these exit channels was similar to that found for the ${ }^{16} \mathrm{O} \rightarrow 4 \alpha$ study.

Monte Carlo simulations of the disassembly of ${ }^{28} \mathrm{Si}$ projectiles have also been started. Figure IIC1 compares the simulated and experimental distributions of relative angles between the alpha particles in the ${ }^{12} \mathrm{C}+4 \alpha$ exit channel. The simulations were performed for prompt decay and sequential evaporation mechanisms. Also a fission simulation was performed where the initial decay step involved the fissioning of the projectile into a ${ }^{16} \mathrm{O}$ and a ${ }^{12} \mathrm{C}$ fragment. This particular division of the projectile was chosen as electrofission and projectile decay studies at lower bombarding energies indicate that it is a very important decay mode for ${ }^{28} \mathrm{Si}$ : much more probable than standard statistical model calculations would predict[1]. In the fission simulation, both the ${ }^{16} \mathrm{O}$ and the ${ }^{12} \mathrm{C}$ fragments each evaporate an alpha particle and the resulting ${ }^{8} \mathrm{Be}$ fragment then decays. Neither of the three simulated relative angle distributions reproduce the experimental data in Fig. IIC1. However, the experimental distribution is intermediate between the sequential evaporation and fission simulation suggesting that both these mechanism may contribute to the ${ }^{12} \mathrm{C}+4 \alpha$ exit channel. Figure IIC2 shows that a combination of $50 \%$ of the sequential evaporation mechanism and $50 \%$ of the fission mechanism reproduces the experimental distribution quite well. Further analysis of the data are needed to substantiate this conclusion. In any case the above example demonstrates the selectivity of the relative angle distributions to the reaction mechanism in these type of studies.

\section{References}

[1] B.R. Fulton and W.D.M. Rae, J. Phys. G16.333 (1990). 


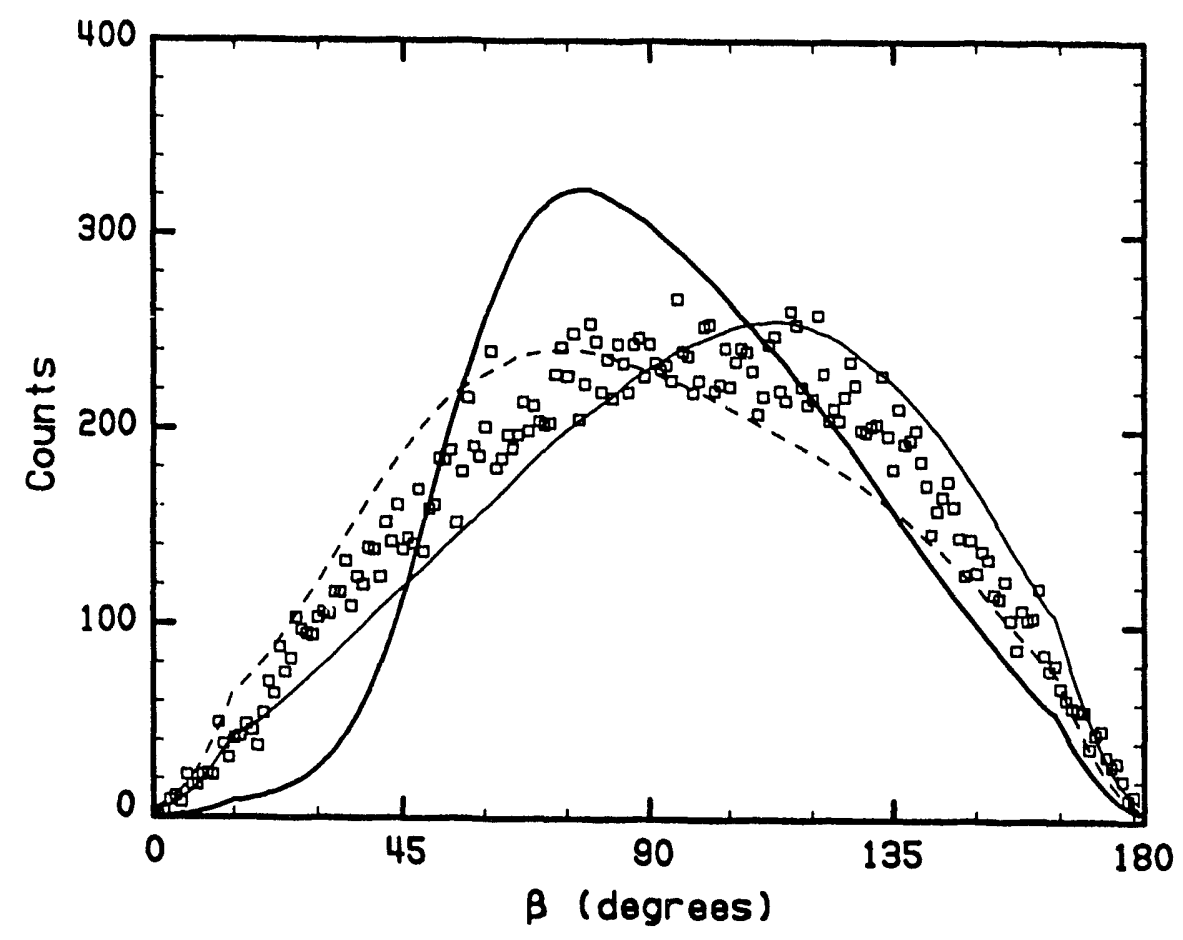

Fig. IIC1: Distribution of relative angles between the alpha particles in the ${ }^{12} \mathrm{C}+4 \alpha$ exit channel. The symbols give the experimental distribution while the thick-solid, thin-solid and dashed lines give the predictions of the prompt, sequential evaporation and fission simulations respectively.

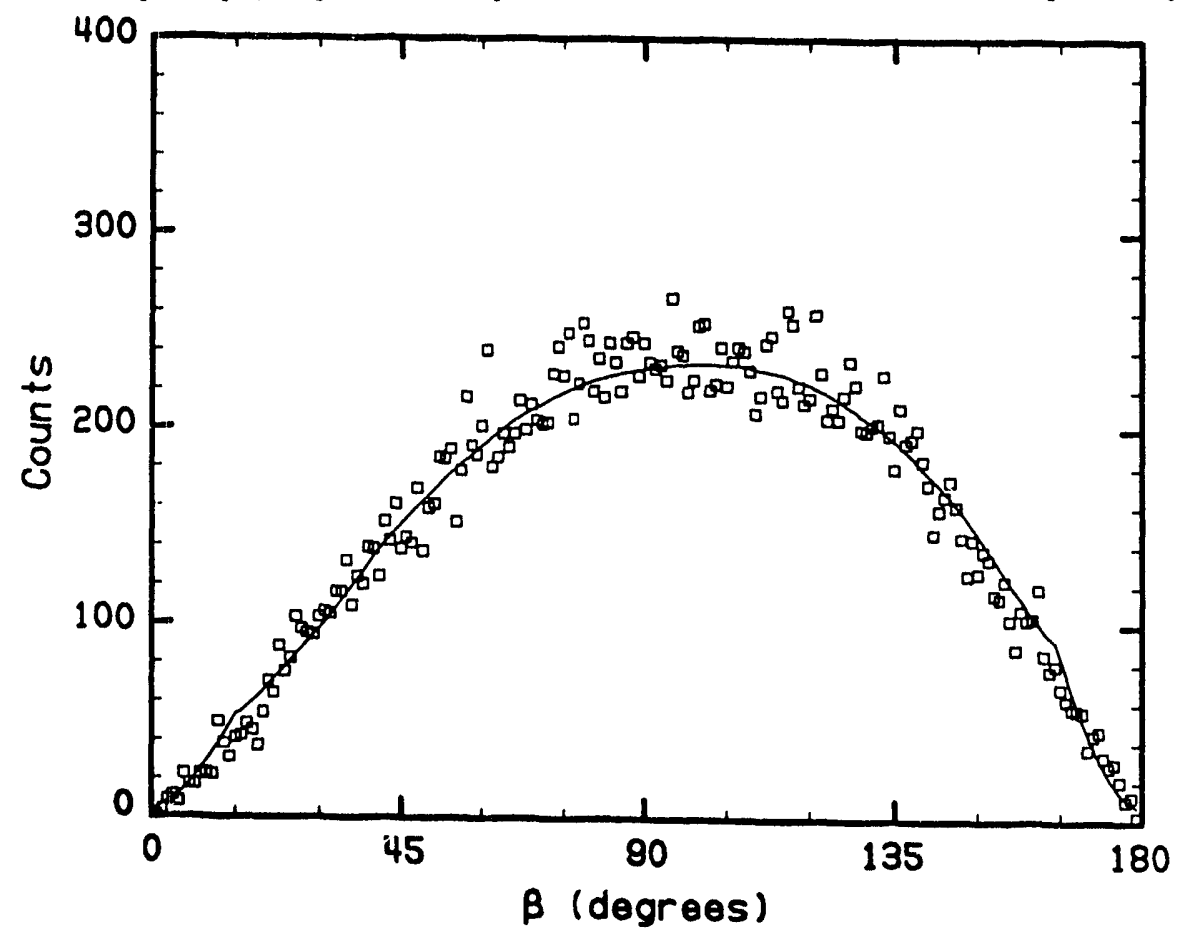

Fig. IIC2: As in Fig. 1, where now the solid line gives the predicted distribution when $50 \%$ of the events are produced by the sequential evaporation mechanism and $50 \%$ from the fission mechanism. 


\section{Large Pre-Fission Multiplicities From Temperature Dependent Friction and Fis- sion Barriers}

\section{R.J. Charity [WU]}

Recent measurements of pre-fission neutron, charge particle and gamma ray multiplicities in heavy ion induced fusion-fission reaction have found unexpectedly large values at high excitation energies compared to predictions made with the statistical model. These measurements indicate that the time scale for the decaying system to reach its scission point is surprisingly large; of the order of $10^{-20}$ to $10^{-19} \mathrm{~s}[1]$. The most popular interpretation of these results is in terms of a model first proposed by Kramers[2] in 1940. Kramers considered the escape of a system across a potential barrier induced by fluctuation along the collective coordinate. The magnitude of the fluctuations is related to the friction associated with these motions and both are due to coupling between the intrinsic and collective degrees of freedom of the system. For Markovian motion the equation of motion along the fission coordinate $\mathrm{x}$ can be described by the Langevin equation:

$$
m \ddot{x}+\frac{\partial V}{\partial x}+\gamma m \dot{x}+\xi(t)=0
$$

where $m$ is the mass associated with fission motion, $V$ is the potential energy, $\gamma$ is the friction and $\xi(t)$ is a fluctuating force resulting from the coupling with the non-fission degrees of freedom. The probability flux can be described by the Fokker-Planck equation for which Kramers found quasi-stationary solutions at high and low friction for a potential energy function composed of connecting upright and inverted parabolas. Kramers found that the quasi-stationary escape rate across the barrier is generally reduced form that predicted with transition state theory. For moderate to strong values of the friction, the Kramers' escape width $\Gamma_{K}$ is related to the transition state theory value $\Gamma_{T S T}$ by

$$
\Gamma_{K}=\frac{\sqrt{\gamma^{2}+4 \omega_{b}^{2}}-\gamma}{2 \omega_{b}} \Gamma_{T S T}
$$

where $\omega_{b}$ is the frequency of the inverted parabolas at the the saddle-point configuration. For moderate friction, the transition state and Kramers' decay are simular. As the friction increases, the Kramers' decay width decreases. Also in the limit of very low friction, Kramers predicts the decay width decreases linearly to zero. In more recent times, Kramers' theory has been extended to include non-Markovian motion and the quasi-stationary decay width has been solved for all values of friction[3]. Kramers' decay rates have been experimentally confirmed in studies of chemical reaction rates[4].

Assuming a constant friction, the inclusion of Kramers' quasi-stationary decay widths into statistical model calculations will not in itself greatly change the predicted pre-saddle neutron multiplicity, but will of course decrease the predicted fission cross section. Now, the Kramers' decay widths are only applicable when the shape degrees of freedom associated with fission are fully equilibrated. If these degrees of freedom are not equilibrated, then the decay rate can be greater or smaller than the equilibrium or quasi-stationary decay rate. A transient fission decay rate has been investigated[5, 6, i] for systems which initially all have the ground state deformation. This assumption implies that systems are not true compound nuclei for which all degrees of freedom are equilibrated. In the case of high friction or over damped motion, 
fission is only possible after some of systems have diffused along the fission coordinate to the saddle point. The fission rate is therefore suppressed approaching the quasi-stationary limit as the deformation degrees of freedom equilibrate. The time interval $\tau_{t f}$ over which the fission is reduced was calculated to be proportional to $\gamma[6,7]$. At a sufficiently high excitation energy such that $\tau_{t f}>\hbar / \Gamma_{l p}$ where $\Gamma_{l p}$ is the decay width for light particle emission, light particle evaporation will occur before the fission decay rate has reached its quasi-stationary value leading to larger pre-saddle multiplicities.

The transient fission rate model can be criticized on two counts. Firstly, it is not clear to what extent the deformation degrees of freedom are not equilibrated and secondly, if these degrees of freedom were not equilibrated it is not clear why all systems will start with the ground state deformation. In this report we investigate to what extent the measured pre-fission multiplicities can be understood without recourse to transient fission widths, but can be compatible with compound nucleus decay albeit with Kramers' quasi-stationary decay rates.

The above discussions have only considered the effects of a constant friction independent of the temperature, shape, mass or charge of the decaying system. The possibility of a temperature dependent friction may offer a solution to explain the large pre-fission multiplicities. If the friction increases fast enough with temperature, the fission decay width will be suppressed at high excitation energies and fission can occur only after the decaying system has evaporated enough light particles, cooling its temperature to point where the fission suppression is reduced. This explanation implies that the fission probability decreases with excitation energy at large excitation energies. To investigate this possibility, statistical model calculations have been performed including a temperature dependent friction. The friction was assumed to vary with temperature as

$$
\gamma=\beta_{1} T \text { or } \gamma=\beta_{2} T^{2}
$$

where $\beta_{1}$ and $\beta_{2}$ are input parameters. Fission probabilities and pre-saddle multiplicities were calculated with a modified version of the statistical mode code PACE where the large friction form of Kramers' fission decay width (Eq. 0.2) was used instead of the transition state value. A total pre-fission multiplicity was obtained by adding on a saddle-to-scission multiplicity to the pre-saddle multiplicity. The saddle-to-scission multiplicity was estimated by allowing the fissioning systems to evaporate light particle for a time interval calculated from Ref. [8]. The decay widths for the fissioning systems were calculated using transmission coefficients for spherical nuclei calculated by PACE. This is not exactly correct, but will suffice to give better than an order of magnitude predictions.

Calculations were performed for two reactions, firstly the reaction ${ }^{18} \mathrm{O}+{ }^{150} \mathrm{Sm}$ at the bombarding energy of $121 \mathrm{MeV}$ forming the compound system ${ }^{168} \mathrm{Yb}$. Fission and evaporation residue cross sections[9] and pre-fission neutron multiplicities[10] have been measured for this react:on. For the low excitation energies of $94 \mathrm{MeV}$ achieved in this reaction, the measured pre-fission neutron multiplicities are similar to pre-saddle multiplicities predicted with standard statistical model calculations although the fission cross section is lower than the predictions. The second reaction for which calculations were performed is ${ }^{16} \mathrm{O}+{ }^{142} \mathrm{Nd}$ which produces the compound nucleus ${ }^{158} \mathrm{Er}$ not too different in mass form ${ }^{168} \mathrm{Yb}$. The pre-fission neutron multiplicity has been measured by Hinde et al. $[1,11]$ at a bombarding energy of $178 \mathrm{MeV}$. The excitation energy is assumed to be $120 \mathrm{MeV}$ after allowing for the emission of one pre-equilibrium neutron[12] and at this higher excitation energy the standard statistical models underpredict the measured pre-fission neutron multiplicities. The fission cross section for this reaction has also been measured[13, 14]. 
Calculation were performed using standard sets of statistical model parameters which have generally been able to reproduce experimental fission excitation functions when Kramers' reduction factors were not used. It is important to determine whether these parameter are still able to reproduce the experimental data when Kramers' decay widths are used in the calculations.

For the ${ }^{168} \mathrm{Yb}$ system, the measurement of both the fission and evaporation residue cross sections enables the fusion cross section to be determined. This in turn allows the maximum angular momentum for fusion $\ell_{c}$ to be deduced. For the ${ }^{158} \mathrm{Er}$ system this is not the case as the evaporation residue cross section has not been measured and the bombarding energy is sufficiently high that low energy fusion models are not reliable for predicting $\ell_{c}$. This parameter is thus undetermined and was adjusted for each input value of $\beta_{1}$ or $\beta_{2}$ to give the experimental fission cross section. For the ${ }^{168} \mathrm{Yb}$ system as $\ell_{c}$ is known, both $\beta_{1}$ and $\beta_{2}$ will be adjusted to try and fit both the measured pre-fission neutron multiplicity and fission cross section simultaneously.

The calculated pre-fission neutron multiplicities and fission cross section for ${ }^{168} \mathrm{Yb}$ system are plotted as a function of $\beta_{1}$ and $\beta_{2}$ in Figs. IID1a to 1c. The pre-saddle neutron multiplicities are indicated by the dashed curves in Fig. IID $1 \mathrm{a}$ and $1 \mathrm{~b}$ while the solid curves indicate the pre-saddle plus saddle-to-scission multiplicities. The hatch band indicate the experimental data. At $\beta_{1} \approx 2 \times 10^{21} \mathrm{~s}^{-1} \mathrm{MeV}^{-1}$, both the measured fission cross section and pre-fission neutron multiplicities are well reproduced by the calculations in Figs. IID1a and 1c. Similarly for $\beta_{2} \approx 1 \times 10^{21} \mathrm{~s}^{-1} \mathrm{MeV}^{-2}$ in Figs. IID $1 \mathrm{~b}$ and $1 \mathrm{~d}$.

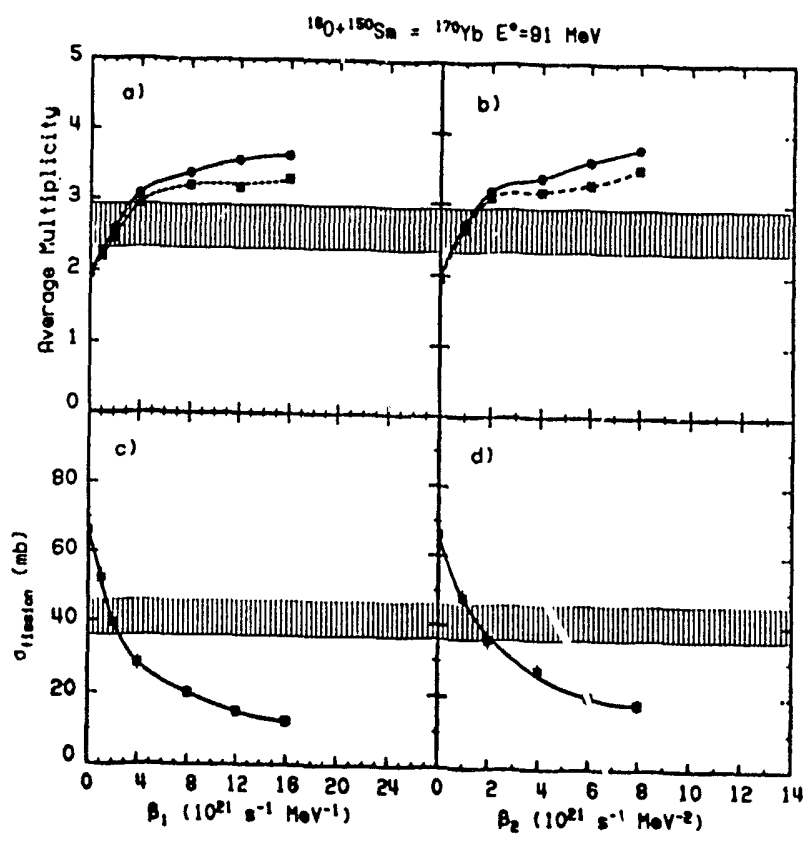

Fig. IID1: Predicted $a, b)$ pre-fission neutron multiplicities and $c, d$ ) fission cross sections obtained assuming $a, c)$ linear and $b, d)$ quadratic dependences of the friction on temperature for the reaction $\mathrm{E}_{l} a b=121 \mathrm{MeV}{ }^{18} \mathrm{O}+{ }^{150} \mathrm{Sm}$. The hatched regions correspond to the experimental quantities with their error. The dashed curves in $a, b$ indicate the pre-saddle and the solid curve the pre-fission multiplicity. 
The calculated pre-fission multiplicities for the ${ }^{158} \mathrm{Er}$ system are shown in Figs. IID2a and 2b. Larger values of $\beta_{1}$ and $\beta_{2}$ are required to reproduce the experimental multiplicity. Thus the inclusion of temperature dependent friction into standard statistical model calculation is not able to reproduce the experimental data unless the friction is also mass dependent or angular momentum dependent.

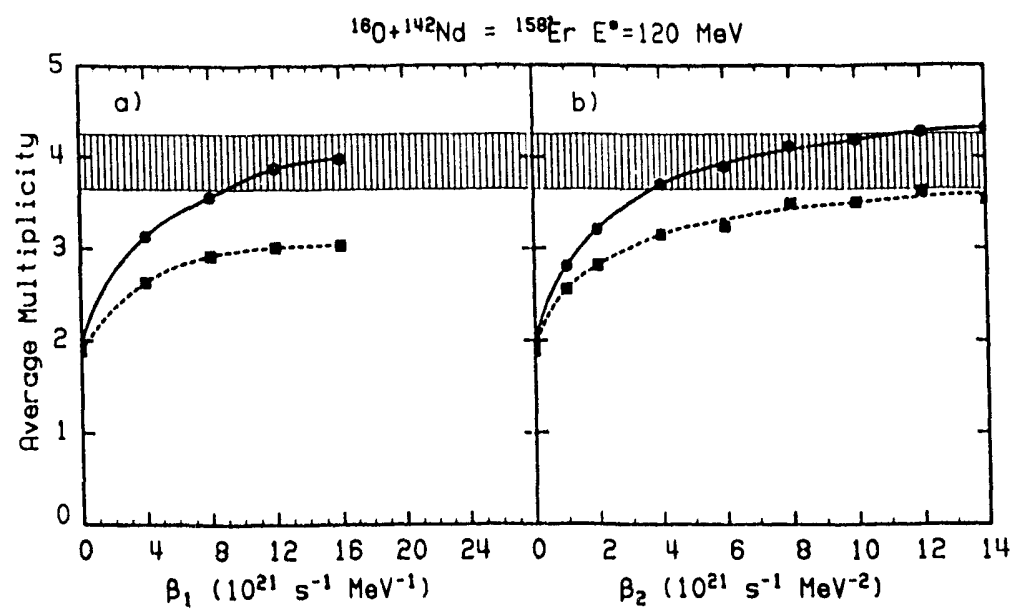

Fig. IID2: Predicted pre-fission neutron multiplicities (solid curve)) obtained for the $\mathrm{E}_{l a b}=178 \mathrm{MeV}$ ${ }^{16} \mathrm{O}+{ }^{142} \mathrm{Nd}$ reaction assuming a) linear and b) quadratic dependences of the frictior on temperature. The hatched region corresponds to the experimental results and the dashed curve indicates the predicted pre-saddle component.

Another predicted effect which is also neglected in most standard statistical model calculations is the decrease of the fission barrier with temperature $[15,16]$. To lowest order, the fission barrier is predicted to decrease quadratically with temperature[16]

$$
B_{f}(T)=B_{f}(0)-k T^{2} \text {. }
$$

The effect of including such a temperature dependence on the level density expression can be shown to be equivalent to using zero-temperature barriers and an effective level density parameter $a_{e f f}=a+k$. Increasing the effective level density parameter at the saddle-point will increase the predicted fission cross section and decrease the predicted pre-fission neutron multiplicities[17]. This compounds the disagreement between standard statistical model calculations and the measured pre-fission neutron multiplicities.

The effect of both both temperature dependent friction and fission barriers have been incorporated into statistical model calculations. The parameter $\mathrm{k}$ in Eq. 0.4 was obtained as a function of angular momentum from fitting the temperature and angular momentum dependent fission barriers for ${ }^{152}$ Dy calculated by Garcias et al.[18].

The predicted pre-fission neatron multiplicities and the fission cross sections for the ${ }^{168} \mathrm{Yb}$ system are plotted in Figs. IID3a to $3 \mathrm{~d}$. The calculated pre-fission neutron multiplicities for 
the ${ }^{158} \mathrm{Er}$ system are shown in Figs. IID $4 \mathrm{a}$ and $4 \mathrm{~b}$. Large values of $\beta_{1}$ or $\beta_{2}$ are required to reproduced the experimental data for both systems. For a $\mathrm{T}^{2}$ dependence of the friction, the two experimental pre-fission neutron multiplicities and the fission cross section for ${ }^{168} \mathrm{Yb}$ system can be all reproduced with a value of $\beta_{2} \approx 1 \times 10^{22} \mathrm{~s}^{-1} \mathrm{MeV}^{-1}$ in Figs. IID3b,3d, and $4 \mathrm{~b}$. This is not to say that the linear temperature dependence of the friction is inconsistent considering the uncertainties in calculating the saddle-to-scission multiplicities. It is interesting to note that the saddle-to-scission component is significantly larger in these calculations than for those without temperature dependent barriers. In fact the predicted pre-saddle neutron multiplicities (dashed curves) in Figs. IID3 and 4 for large values of either $\beta_{1}$ or $\beta_{2}$ are very similar to the predictions obtained without friction and temperature dependent barriers (see Figs. IID1 and 2 at $\beta_{1}, \beta_{2}=0$ ). The opposite effects of the temperature dependent fiction and fission barriers have largely cancelled. Future calculations must determine whether this is true for heavier and lighter compound nuclei and whether the inclusion of temperature dependent friction and fission barriers can reproduce the systematics of pre-fission neutron multiplicities and fission cross sections.

\section{References}

[1] D.J. Hinde et al., Phys. Rev. C39, 2268 (1989).

[2] H.A. Kramers, Physica 7, 284 (1940).

[3] E. Pollak, H. Grabert, and P. Hänggi, J. Chem. Phys. 91, 4073 (1989).

[4] P. Hänggi, P. Talkner, and M. Borkovec, Rev. Mod. Phys. 62, 251 (1990).

[5] P. Grangé and H.A. Weidenmüller, Phys. Letts. B96, 26 (1980).

[6] P. Grangé, J.Q. li, and H.A. Weidenmüller, Phys. Rev. C27, 2063 (1983).

[7] K.H. Bhatt, P. Grangé, and B. Hiller, Phys. Rev. C33, 954 (1986).

[8] H. Hofmann and R.J. Nix, Phys. Letts. 122B, 117 (1983).

[9] R.J. Charity et al., Nucl. Phys. A457, 441 (1986).

[10] D.J. Hinde et al., Nucl. Phys. A452, 5:50 (1986).

[11] D.J. Hinde et al., Phys. Rev. C37, 292:3 (1988).

[12] A. Gavron et al., Phys. Rev. C35, 579 (1987).

[13] J. van der Plicht et al., Phys. Rev. C28, 2022 (1983).

[14] A. Gavron et al., Phys. Rev. C30, 1550 (1984).

[15] R.W. Hasse and W. Stocker, Phys. Letts. B44, 26 (1973).

[16] G. Sauer, H. Chandra, and U. Mosel, Nucl. Phys. A264, 221 (1976).

[17] D.Ward et al., Nucl. Phys. A403, 189 (1983).

[18] F. Garcias et al., Z. Phys. A336, 31 (1990). 
${ }^{18} 0+{ }^{150} \mathrm{Sm}=170 \mathrm{Yb} \mathrm{E}^{\bullet}=91 \mathrm{MeV}$

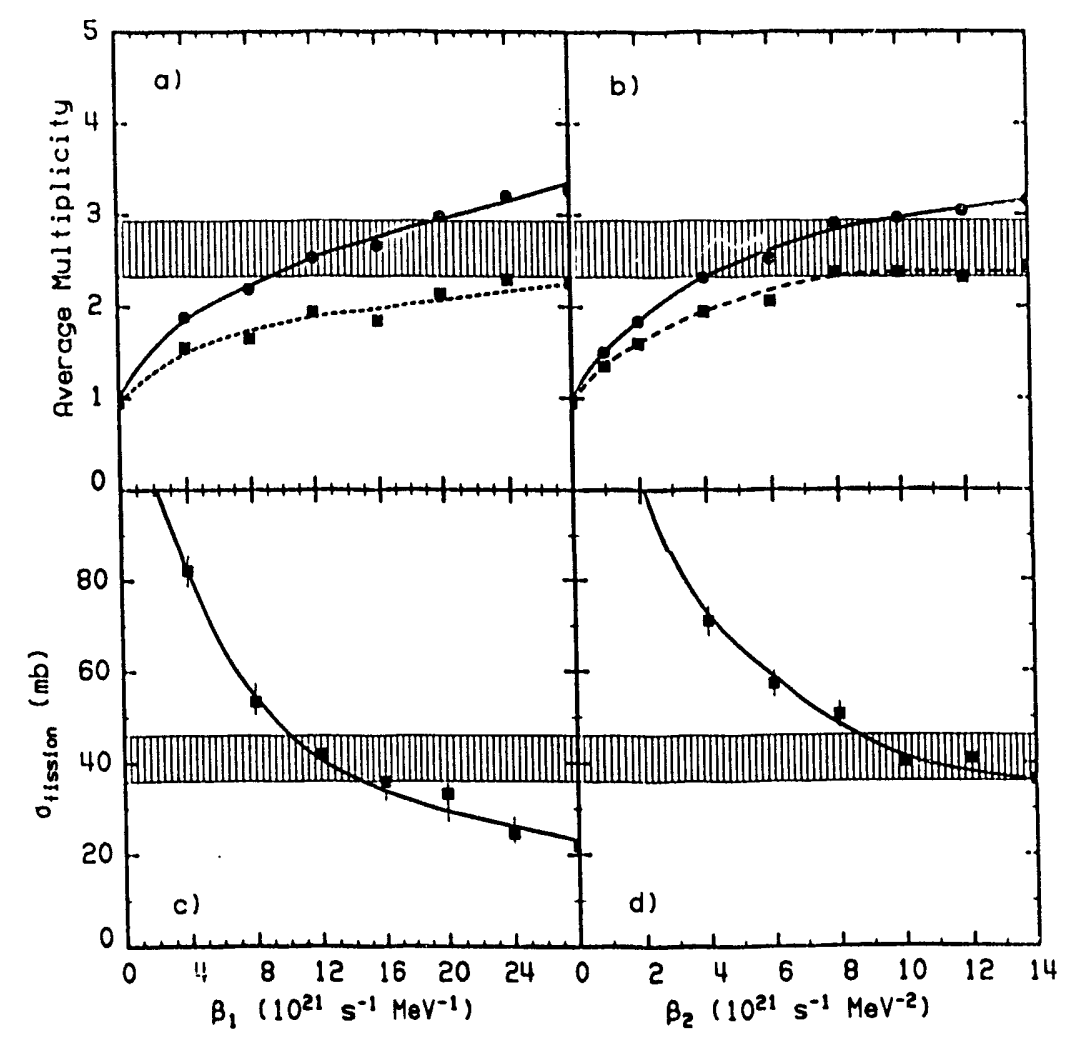

Fig. IID3: As for Fig. 1, now when temperature dependent fission barriers are included in the calculations.



Fig. IID4: As for Fig. 2, now when temperature dependent fission barriers are included in the calculations. 


\section{E. Particle Multiplicity Correlations}

S.P. Baldwin, B. Lott, B.M. Quednau, B.M. Szabo, W.U. Schröder, J. Töke [ROCH], J. Barreto, R.J. Charity, L. Gallamore, D.G. Sarantites, L.G. Sobotka [WU], and R.T. de Souza [MSU]

Event characterization in the intermediate energy domain has evolved from few particle measurements to the so called $4 \pi$ measurements. The $4 \pi$ measurements have either involved neutrons or charged particles. However to obtain a complete gobal characterization of the event, both neutrons and charged particles must be detected with high efficiency. We have recently completed the first of such measurements. Ultimately such studies will provide the most stringent tests of models dealing with the formation and decay of highly excited nuclei. These studies must answer question of whether the particle production observed in these reactions can only be explained by dynamical models which proceed through a bulk density reduction in the interaction region. We will not, in this report, discuss this issue. (The reader will have to wait for the next issue of our progress report.) However, we will briefly present some the the preliminary results of what is the most complete study of the final products of an intermediate energy reaction.

The reaction products from reactions of $28.2 \mathrm{MeV} / \mathrm{u}{ }^{136} \mathrm{Xe}+{ }^{207} \mathrm{Bi}$ where studied with the Dwarf system (Ball and Wall) and the University of Rochester Neutron Multiplicity Meter (NMM). The Dwarf system provided the energy and coarse position information for light ions ( $\mathrm{p}, \mathrm{d}, \mathrm{t},{ }^{3} \mathrm{He}, \alpha$, and heavy ions) which the NMM provides a reasonably high efficiency $(\approx$ $75 \%)$ measure of the number of neutrons emitted from slow sources. The first correlation to be extracted from these data is that between the neutron multilplcity $\left(m_{n}\right)$ and the gross overall charged particle multiplicity $\left(\mathrm{m}_{z}\right)$, see Fig. IIE1. The great majority of the events are peripheral. These events exibit a very large range of neutron mutiplities while the associated low charged particle multiplicities are extremely small. For more central collisions, the charge particle multiplicity as well as the neutron multiplicity provide measures of the violence. Therefore, at least for the heavy neutron laden system of the present study, only neutrons can be used as a violence selector or as a crude impact paramenter selector throughout the full range of impact parameters. This suggests that a neutron multiplicity plus high energy photon study, analogous to the charged particle plus high energy photon study discussed in section $\mathrm{A}$, might provide valuble information on particle multiplicity fluctuations. 




Fig. IIE1: Contour plot of the charged particle multiplicity versus the neutron multiplicity. The lines are calculated correlations between free neutron production and production of charged particles of various types. 


\section{F. Molecular Beam induced Fusion.}

W. Garver, J. J. Leventhal [UMSL], and L. G. Sobotka [WU]

Recent work at Brookhaven by R. Beuhler, G. Friedlander, and L. Friedman has studied d + $\mathrm{d}$ fusion in collisions of large ice clusters on solid targets with high deuterion densities. $[1,2,3,4]$ They argue strongly that there is an enhancement in the $d+d$ fusion yield in this type of collision over that from collisions between individual $d+d$ collsions. The explanation that is offered is that the cluster collisions create a small region, or spike, in the target of high energy density and that this hot spike survives sufficently long to develop the Boltzmann-like tail on the ion energy distribution. This being so, a Gamow type calculation is justified and therefore the mean energy is not the relevant quantity, but rather, the time development and decay of the high kinetic energy portion of the ion energy distribution in the thermal spike. Beuhler et al. further argue that the fusion is fostered by the fact that the rate of energy loss $(\mathrm{dE} / \mathrm{dX})$ of the cluster in the target material at the energies of their study is dominated by nuclear stopping. ${ }^{[7]}$ This promotes rapid development of the kinetic Boltzmann tail because energy is pumped directly into motion of the nuclei. (It is perhaps worthy of noting that laser confinement fusion suffers from the opposite situation. Energy is pumped into, and thus to a large extent lost, by energy depostion in electron current waves or plasmons.) The importance of the work done at Brookhaven is not only to study this new path to low energy light element fusion, but also to study the nonequilibrium aspects of the energy transport in finite systems. In fact the energy transport issues are the

most interesting. This point is made quite clear by collision simulations $[5,6]$, (which include both reasonable energy transport dynamics and, most importantly, the contribution of knock-on reactions to the fusion yield) are not wble to reproduce the cluster size dependence of the fusion yield observed by the Brookhaven group. Since the initial diameter of the hot spike will only vary with the $1 / 3$ power of the number of units in the cluster, studies with vastly different sizes are important. This motivates our project as well as the need for futher verivication of the Brookhaven results. We plan to study the $d+d$ fusion driven by the impact of molecular ions with targets with a high density of deuterons. We have no data to present only progress on the experimental facility required to conduct this work.

We have constructed a small molecular beam accelerator in the Atomic Physics laboratory at UMSL. The accelerator consists of a hot cathode ion source with three lenses which sits on a high voltage platform, an acceleration stack (from the terminal with negative high voltage to ground), a Wien filter (crossed electric and magnetic fields), a second electric field region, and finally a chamber with a target on a vacuum interlock and a solid state Si detector to detect the fusion products, see Fig. IIF1. The Wein filter is a velocity, or equilivantly, a mass selector. The second electric field, puts a small bend in the beam line, which we have found to be essential for removing neutral beam species which after acceleration, but before passing the filter, have recombined with an electron. The voltage source is a $1 \mathrm{ma}, 300$ Kilovolt Glassman high voltage supply. It was purchased jointly by Washington University (not from funds from this grant) and the University of Missouri in St. Louis.

Wo are presently testing all elements of this system. These tests include: the optical control system for the tuning of the source which sits on the high voltage platform, mass resolution tests of the beam transport system, high voltage tests, and detection efficiency measurments using $\mathrm{d}+\mathrm{d}$ reactions at low ( $<100 \mathrm{keV}$ ) energy: The optical system is operational (it controls $\mathrm{DC}$ to $\mathrm{DC}$ transformers which run off of a car battery which sits on the high voltage platform). We 
have achieved better than $3 \%$ mass resolution with the present filter. We are presently moving from an acceleration stack and vacuum vessel which prevented us from going over $100 \mathrm{KV}$ to one which we hope will ultimately take us io near $300 \mathrm{KV}$.

\section{References}

[1] R.J. Beuhler, G. Friedlander, L. Friedman, Phys. Rev. Lett. 63,1292(1989).

[2] R.J. Beuhler and L. Friedman, Chem. Rev. 86,521(1986).

[3] R.J. Beuhler, Y. Y. Chu, G. Friedlander, L. Friedman, J. G. Alessi, V. LoDestro, J. P. Thomas, Phys. Rev. Lett. 67, xxxx(1991).

[4] R.J. Beuhler, G. Friedlander, L. Friedman, submitted to Accts Chem. Res. (1991).

[5] C. Carraro, B. Q. Chen, S.Schramm, and S. E. Koonin, Phys Rev. A42. 1379 (1990).

[6] M. H. Shapiro and T. A. Tombrello, Phys, Rev. Lett. 65, 92 (1990).

[7] J. Ziegler, Computer code TRIM91.

[8] N. Jarmie, Los Alamos National Laboratory Report LA-UR-84-3349, 1984, unpublished. 


\section{Molecular Beam Apparatus}

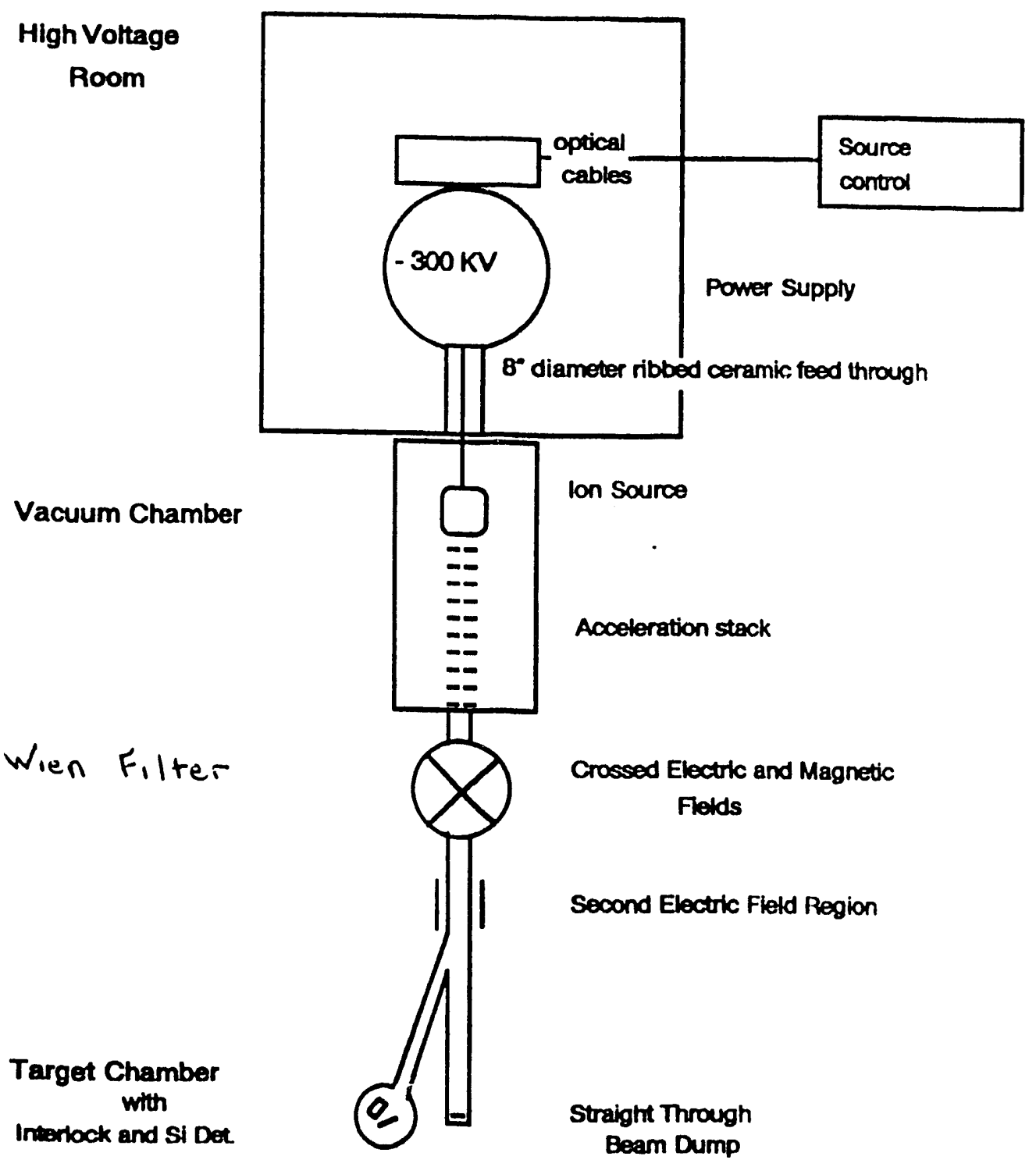

Fig. IIF1: Molecular beam apparatus. 


\title{
III. TECHNICAL
}

\section{A. Dwarf Ball and Wall (DBW)}

\author{
D.G. Sarantites, P. F. Hua, J. Elson, R.J. Charity, and L.G. Sobotka [WU]
}

The jwarf Ball and Wall (DBW) system is well suited for low energy reaction studies and as an aid to spectroscopy studies. We suspect that the overlap area between these two fields may very well turn out to be the field of study where this device can make the most significant contibutions. We therefore are interested in improving the qualities of the DBW most appropriate for these low energy studies. To this end changes are being made in both the detection hardware and the electronics. This effort is being spearheaded by D.G. Sarantites and P. F. Hua.

The primary change to the detection hardware is to construct a set of thin $(2 \mathrm{~mm})$ CsI detectors. Our original plan was to use these thin detectors in front of intrinsic Ge detectors, used in most of our low energy projects, in order iu reduce photon absorbtion. A recent experiment suggests that an additional problem, Compton scattering off of the CsI which introduces a background in Ge detectors, can also be reduced by thiner CsI detectors. However, because this problem was found to have a significant large angle component, just reducing the thickness of the detectors in front of the Ge's is inadequate. Reducing the thinkness of additional detectors or even changing to fast-slow plastic phoswich elements are possible options.

On the electronics side, we believe that considerable progress can be made in improving the high count rate performance of the Dwarf system. Presently, the SLOW (integration of the current pulse from 0-400 ns) and the TAIL (1500-3000 ns) parameters are used for light-ion identification. An alternative which while providing the required pulse shape discrimination, will be able to discriminate against true pile-up pulses is to use the SLOW and the digitized time over the discriminator threshold. The version of the discriminators which we have been using for the last few years has individual ECL outputs which have widths equal to the time over the threshold. The recent purchase (from Sarantites' grant) of the new LeCroy 2277 Multihit TDC will enable us to test this technique. This 32 channel real time TDC can register 16 hits per channel which may be cither the negative or positive going edges of the signal. The time over threshold can be directly determined from the time difference between the first two hits, ( the first negative going the second positive going). Subsequent hits will be due to recrossings of the threshold level. These recrossings will be due to either statistical fluctuations of the original signal (these will be of short duration) or due to pile-up of another pulse. Therefore the use of this new TDC will not only provide a new method of pulse shape discrimination by which charge particles can be identified, but also it will also provide a pile-up rejector. 


\section{B. Mini Wall (MW)}

L. Gallamore, L. G. Sobotka, J.T. Hood, J. Elson, R.J. Charity, and D.G. Sarantites [WU]

The intermediate energy reaction studies done by our group require greater granularity in the forward direction than is offered by the Dwarf system. Oddly enough the MSU MINIBALL system suffers from the same inadequacy. The newer MINIBALL system is however in most other regards ideally suited to the study of intermediate energy heavy-ion reactions. In order to improve our capability for the study of these complex reactions and to remove the one major definciency of the MINIBALL system, we have construted a forward highly granular wall which mates to the MSU system. We call this device the MINI WALL and it is shown in Fig. IIIB1. It consists of 128 fast plastic-CsI(Tl) phoswich elements. Many of the important specifications of this device are provided in Table IIIB1.

By maintaining the same technolgy as the Dwarf system we only needed to make one significant change to the electronics used to run the Dwarf system. This change is replacement of the LeCroy FERA ADC's, which have a common gate for each module ( 16 channels), with a Phillips $7167 \mathrm{ADC}$, which have individual gates. This change is made necessary by the larger distances between the target and the detectors and thus a larger variation in the fight time of the ions to the detectors. These ADC's are presently being evaluated by our group as well as by our collaborators at MSU.

A significant improvment over the Dwarf device, in-so-far as the preformance of this device for intermediate energy reaction studies, is in the pulse shape discrimination (PSD) of the CsI for ligh-ion identification. Studies at MSU suggested that a higher Tl dopant concentration extended the PSD beyond isotope identification for charge one and two. The crystals (purchased from Hilger Analytical) used in the MINI WALL have Tl doping in the range of $1550-1900 \mathrm{ppm}$. The improved PSD was demonstrated in a recent test run at MSU. Figure IIIA2 shows a two dimensional map of the SLOW parameter (resulting from the integration of the current from 0 $400 \mathrm{~ns})$ versus the TAIL parameter (1500ns - $3000 \mathrm{~ns})$. In addition to the light-ion identification ( $\mathrm{p}, \mathrm{d}, \mathrm{t},{ }^{3} \mathrm{He}$,and $\alpha$ ) these crystals also provide for the identification of the isotopes of $\mathrm{Li}$ and the elements $\mathrm{Be}$ and $\mathrm{B}$.

This project has been spearheaded by the efforts of Larry Gallamore, R. J. Charity, and L.G. Sobotka with the assistance of every living body in the WU nuclear chemistry group and a few souls from MSU.

Table IIIB1: Mini Wall Characteristics

\begin{tabular}{||r|r|r|rr|rrr||}
\hline Ring & $\begin{array}{r}\text { No. of } \\
\text { Det. }\end{array}$ & $\begin{array}{r}\text { interface } \\
\text { area }\left(\mathrm{cm}^{2}\right)\end{array}$ & $\begin{array}{r}\text { solid } \\
\mathrm{msr} / \mathrm{det}\end{array}$ & $\begin{array}{r}\text { angle } \\
\mathrm{msr} / \mathrm{ring}\end{array}$ & \multicolumn{3}{|c||}{ theta(deg) } \\
center & high \\
\hline 1 & 16 & 2.832 & 1.04 & 16.7 & 3.28 & 4.32 & 5.36 \\
2 & 16 & 2.668 & 2.45 & 39.2 & 5.44 & 6.95 & 8.55 \\
3 & 22 & 2.622 & 2.40 & 52.9 & 8.56 & 10.00 & 11.44 \\
4 & 26 & 2.896 & 2.66 & 69.1 & 11.56 & 13.00 & 14.44 \\
5 & 24 & 5.780 & 5.30 & 127.2 & 14.56 & 16.62 & 18.69 \\
6 & 24 & 11.297 & 10.36 & 2.18 .6 & 18.80 & 21.88 & 24.95 \\
\hline
\end{tabular}




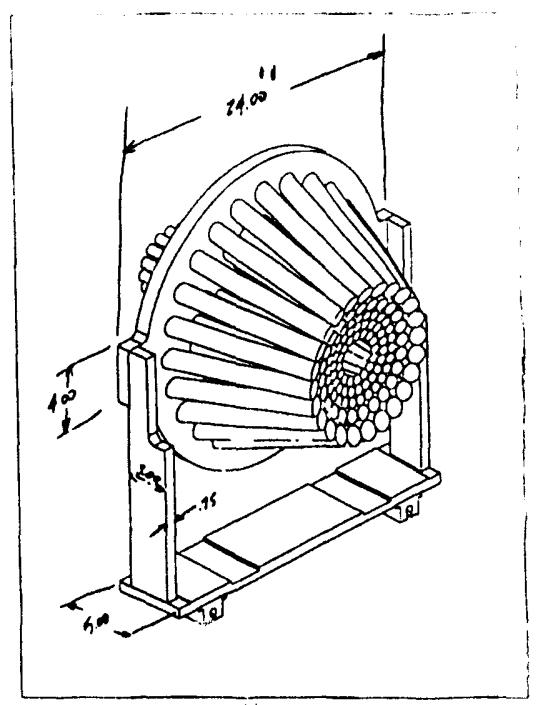

Fig. IIIB1:ABOVE Schematic of the Washington University Mini Wall. Many of the characteristics of this device are summarized in Table IIIB1.

Fig. IIIB2:BELOW Map of the SLOW (abscissa) versus TAIL (ordinate) signals from a MINIWALL detector.

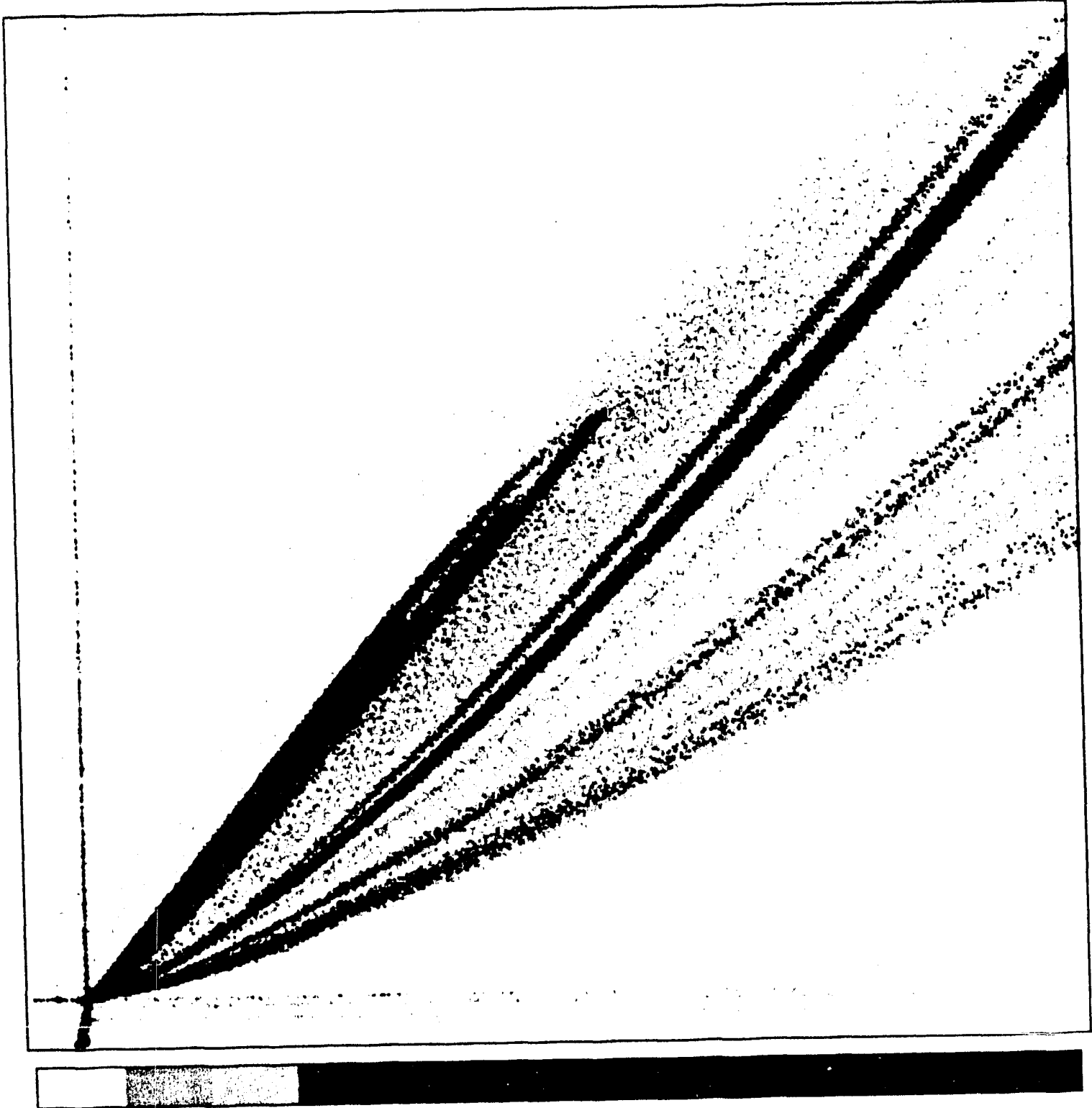




\section{Computer and Local Data Acquisition Systems}

\section{J. Elson, D.G. Sarantites, R.J. Charity, and L.G. Sobotka [WU]}

The Washington University nuclear chemistry group's computer system is a self sufficient network corsisting of four $3100 \mathrm{VAX}$ workstations (two model 76 , one model 45 , and one model 30 ), over 3 Gbytes of disk, five 2.3 Gbyte exabyte $8 \mathrm{~mm}$ tape systems, one streaming real tape drive, and several PC's. Each workstation has at least 16 Mbytes of core. None of these devices have been purchased on this DOE grant! Our contibutions (including one model 76 workstation, most of the disk drives, one exabyte drive, and a portable $386 \mathrm{PC}$ ) were purchased with funds from LGS's PYI award.

Our local data acquisition system is CAMAC based and uses a Kinetic Systems 3922 parallel bus crate controller interfaced to a PC via a KS 2926-21A. Again, these units were purchased from funds available from LGS's PYI award. Additional testing is done on MCA's purchased by Washington University for the radiochemistry teaching laboratory.

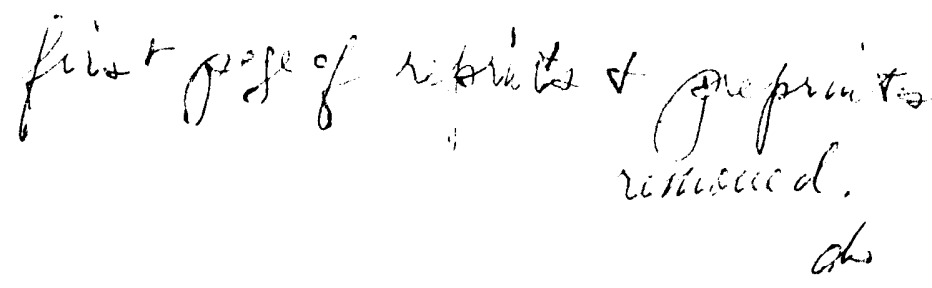



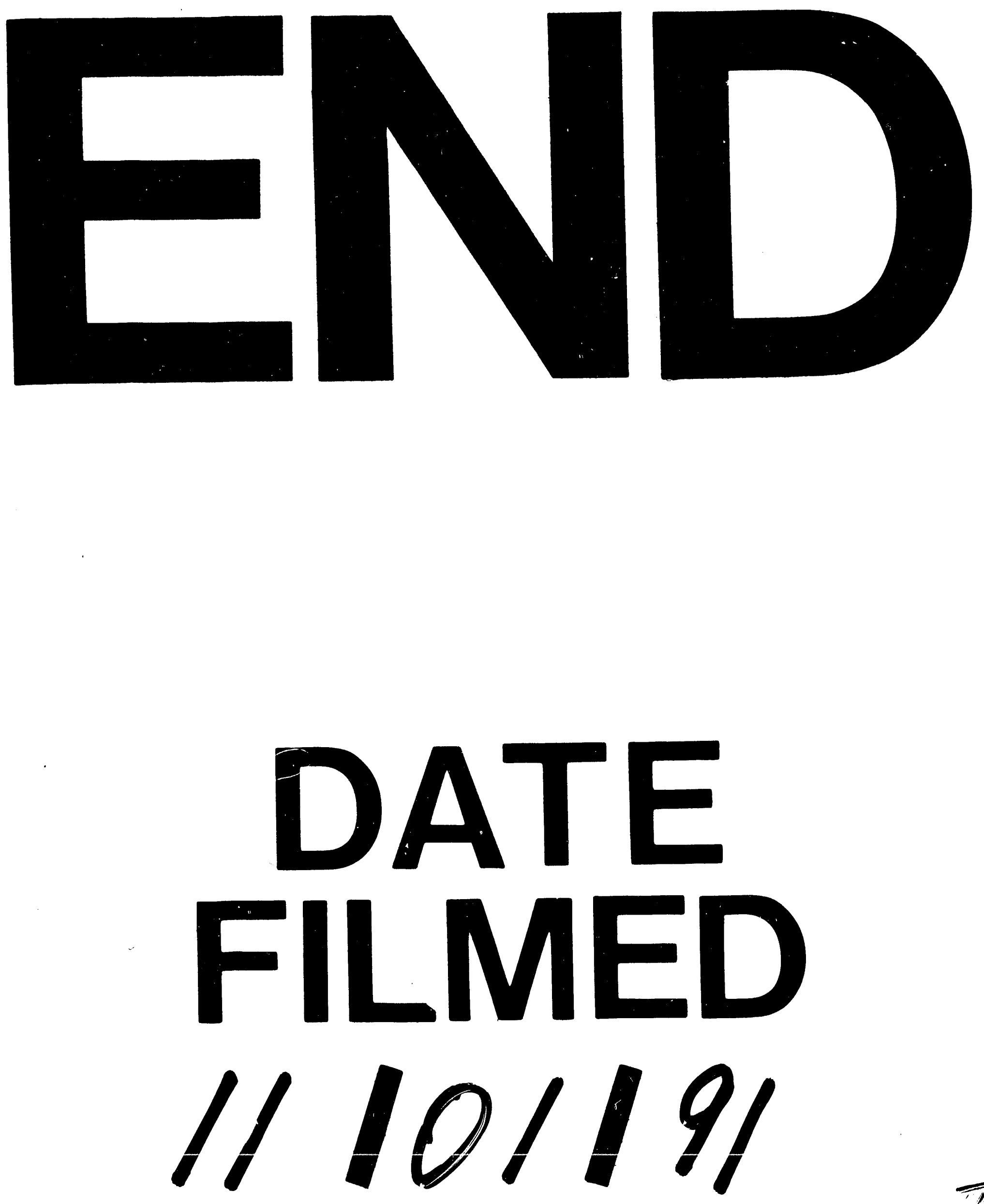

II 
